\title{
GLOBAL REGULARITY AND SCATTERING FOR GENERAL NON-LINEAR WAVE EQUATIONS II. $(4+1)$ DIMENSIONAL YANG-MILLS EQUATIONS IN THE LORENTZ GAUGE
}

\author{
JACOB STERBENZ
}

\begin{abstract}
We continue here with previous investigations 11] on the global behavior of general type non-linear wave equations for a class of small, scaleinvariant initial data. In particular, we show that the $(4+1)$ dimensional YangMills equations are globally well posed with asymptotically free behavior for a wide class of initial data sets which include general charges. The method here is based on the use of a new set of Strichartz estimates for the linear wave equation which incorporates extra weighted smoothness assumptions with respect to the angular variable, along with the construction of appropriate micro-local function spaces which take into account this type of additional regularity.
\end{abstract}

\section{INTRODUCTION}

The goal of this paper is to give a proof and description of the global regularity properties of a wide class of non-linear wave equations on $(4+1)$ dimensional Minkowski space. This is a continuation of our previous work 11]. All the equations we shall consider here are semi-linear wave equations with derivative non-linearities. The generic form for such an object can be written as follows:

$$
\square \phi^{I}=\mathcal{N}\left(\phi^{I}, \nabla \phi^{I}\right),
$$

where $\square=-\partial_{t}+\Delta_{x}$ is the usual D'Lambertian with $\Delta_{x}=\partial_{1}^{2}+\partial_{2}^{2}+\partial_{3}^{2}+\partial_{4}^{2}$ the Laplacean on $\mathbb{R}^{4}$. Here, the superscript notation in the $\phi^{I}$ denotes that we may be considering a system of equations, where the $I$ can be thought of as an index. The non-linearity $\mathcal{N}$ on the right hand side of (1) is some function of $\phi^{I}$ and its first partial derivatives, collectively denoted by $\phi^{I}$. However, we do not allow $\mathcal{N}$ to contain second order derivatives of $\phi$. Also, we will restrict ourselves here to the case where the non-linearity $\mathcal{N}$ has constant coefficients and is a polynomial of degree 2 or higher in the vector $\left(\phi^{I}, \nabla \phi^{I}\right)$ with no interaction of the type $\phi^{2}$. This is not a severe restriction because it still includes the class of gauge-field equations on Minkowski space, which is one of the main motivations of this work:

Specifically, let $(G, \mathfrak{g})$ be a compact, semi-simple Lie group with Lie algebra $\mathfrak{g}$. For a given set of $\mathfrak{g}$-valued functions on Minkowski space $\left\{A_{\alpha}\right\}, \alpha=0, \ldots, 4$, we form the curvature 2 -form:

$$
F_{\alpha \beta}=\partial_{\alpha} A_{\beta}-\partial_{\beta} A_{\alpha}+\left[A_{\alpha}, A_{\beta}\right] .
$$

Then $F$ is said to satisfy the Yang-Mills equations if:

$$
D^{\beta} F_{\alpha \beta}=0,
$$

The author was supported in part by an NSF postdoctoral fellowship. 
where $D_{\alpha} F=\partial_{\alpha} F+\left[A_{\alpha}, F\right]$ denotes the gauge covariant derivative of $F$ in the direction of $\partial_{\alpha}$. Expanding out the equation (3) in terms of the gauge potentials $\left\{A_{\alpha}\right\}$, we arrive at the following second order system of PDE:

$$
\square A_{\beta}=\partial_{\beta} \partial^{\alpha} A_{\alpha}-\left(\left[\partial^{\alpha} A_{\alpha}, A_{\beta}\right]+\left[A_{\alpha}, \partial^{\alpha} A_{\beta}\right]+\left[A^{\alpha}, F_{\alpha \beta}\right]\right) .
$$

If we now make the a-priori assumption that:

$$
\partial^{\alpha} A_{\alpha}=0,
$$

the so called Lorentz gauge condition, then (4) reduces to:

$$
\square A_{\beta}=-\left[A_{\alpha}, \partial^{\alpha} A_{\beta}\right]-\left[A^{\alpha}, F_{\alpha \beta}\right] .
$$

In fact, it turns out that if $\left\{A_{\alpha}\right\}$ is a solution to (6) such that at $t=0$ one has the gauge condition:

$$
\partial^{\alpha} A_{\alpha}(0)=0 \quad \partial^{\alpha} \partial_{t} A_{\alpha}(0)=0,
$$

then (5) is satisfied for all times where the solution in sufficiently smooth. That is, the Lorentz gauge condition propagates. Notice that if the second condition in (7) is to be satisfied, then from the equations (6), we must have that the temporal potential $A_{0}$ satisfies the following elliptic constraint equation at $t=0$ :

$$
\Delta_{x} A_{0}(0)=\partial^{i} A_{i}(0)+\left[A_{\alpha}, \partial^{\alpha} A_{\beta}\right](0)+\left[A^{\alpha}, F_{\alpha \beta}\right](0) .
$$

This implies that in general, the initial data for the system (6) (and indeed for the system (4) ) cannot decay better than $r^{-2}$. This fact is known as the charge problem, and causes certain difficulties in the global theory of (3). We will discuss this in more detail shortly.

We now return to the more general discussion of equations of type (1). Our main concern will be the global in time regularity properties for these kind of systems. This type of question has been considered by many authors for various spatial dimensions, and it is not possible to give here a complete account of all the progress that has been made to date. In the case of 4 spatial dimensions, that is $(4+1)$ dimensional Minkowski space, the first general theory of the global behavior of non-linear systems of the form (10) was given by the breakthrough work of S. Klainerman [3]. Specifically, he showed that if the non-linearity on the right hand side of (11) is schematically of the form:

$$
\square \phi^{I}=\left|\nabla \phi^{I}\right|^{2},
$$

and the Cauchy data:

$$
\phi^{I}(0)=f^{I}, \quad \partial_{T} \phi^{I}(0)=g^{I},
$$

is sufficiently smooth and decays sufficiently fast at (space-like) infinity, then as long as the corresponding norms are small enough a global solution to (9) with this initial data exists. The method of that paper was based on controlling the $L^{\infty}$ norm $\nabla \phi$ to such an extent that Duhamel's principle could be used globally in time. That is, one makes crucial use of the uniform decay of the solutions to the nonlinear problem (9). This decay is provided by certain weighted energy estimates which can naturally be recover by commuting the various weights (vector fields) with the linear equation on the left hand side of (9). At the outset, the result of 
3] did not include the case where the non-linear system (11) takes the form (again schematically):

$$
\square \phi^{I}=\phi^{I} \nabla \phi^{I},
$$

which is the general interaction type of the Lorentz Yang-Mills equations (6). A somewhat more involved argument is needed to get around the fact that the natural quantity one can gain $L^{\infty}$ control of via the energy method is $\nabla \phi^{I}$ instead of $\phi^{I}$. This problem was handled by Hörmander in [1, who used certain Riesz potential modifications of the usual energy to gain the needed $L^{\infty}$ control on $\phi^{I}$. Notice that in some sense, the problem (111) is critical with respect to the decay of $\phi^{I}$. This can easily be seen by integrating the naive asymptotic one would have for $\nabla \phi^{I}$ :

$$
\left|\nabla \phi^{I}\right| \sim \frac{\text { const. }}{(t+r)^{\frac{3}{2}}(|t-r|+1)^{\frac{1}{2}}}
$$

to obtain $\left|\phi^{I}\right| \sim t^{-1}$, which just fails to be integrable globally in time. ${ }^{1}$ Heuristically, this means that one cannot close a boot-strapping argument for the system (11) without allowing either modifying the energy in some way, allowing it to grow, or finding some family of exact space-time integrals to control error estimates which come from the differentiation of the non-linearity. A major drawback of the results [3] -1] is that they assume the initial data (10) is either compactly supported or decays in such a way that it is $L^{2}$. That is, the data is assumed to decay like $r^{-2-\epsilon}$ as $r \rightarrow \infty$. As we have mentioned in our previous discussion of the Yang-Mills equations (3), this kind of decay rate is not quite attainable. ${ }^{2}$ For these reasons, as well as its intrinsic interest, we introduce here a completely different method for studying the global behavior of (111) which is based on recent advances in the low regularity theory of general non-linear wave equations. As a point of comparison, this method allows us to handle initial data which only decays like $r^{-1-\epsilon}$ at infinity.

The method we employ here is not based in any way on the uniform properties of solutions to the system (11). Instead, our point of departure will be the following simple observation: Let $\phi^{I}$ be a given solution to the system (11). Then is is easy to see that if one performs the scale transformation:

$$
\phi_{\lambda}^{I}(t, x)=\lambda \phi^{I}(\lambda t, \lambda x),
$$

the resulting function $\phi_{\lambda}^{I}$ is also a solution to the set of equations (11). This is just a reflection of the fact that the equations (11) are homogeneous. Suppose now that one could produce a Banach space $B$ which is dimensionless with respect to the scale transformation (12) at time $t=0$. That is, one has the identity:

$$
\left\|\left(\phi^{I}(0), \partial_{t} \phi^{I}(0)\right)\right\|_{B}=\left\|\left(\phi_{\lambda}^{I}(0), \lambda\left(\partial_{t} \phi^{I}\right)_{\lambda}(0)\right)\right\|_{B} .
$$

\footnotetext{
${ }^{1}$ Of course one can obtain the correct decay directly for $\phi^{I}$ through the use of Morawetz type multipliers. However, the price one pays for this is the presence of extra weights in the energy integral. Once these are taken into account, one will see again that (at least alone the forward light cone $\mathrm{t}=\mathrm{r})$ the decay in $(t+r)$ is critical.

${ }^{2}$ However, note that one is only off by logarithmic divergence. It is likely that this problem can be overcome by using an appropriate fractionally weighted modification of the usual vector-field method. We will not pursue these ideas here, as our approach is much more general and includes initial data sets which decay at a rate that would be highly singular to any straight forward modification of the vector-field technique.
} 
Suppose that furthermore, one had an existence theorem which said that any set of initial data for which (13) is small enough, there is a local in time solution to (11) with this initial data. Then, by simply re-scaling, such a local existence theorem would necessarily be global in time. Because the equations we are considering are hyperbolic, it is natural to look for a $B$ which is an energy type space. A simple calculation shows that on $\mathbb{R}^{4}$, the Sobolev space which is scale invariant with respect to (12) (at $t=0$ ) is the energy space $\dot{H}^{1}$. However, it is not at all unreasonable to expect that such a space is far too weak to control solutions to (11) locally in time. For example, $\dot{H}^{1}$ in 4 spatial dimensions is a whole derivative (and then some) away from controlling $L^{\infty}$. In fact, one can see immediately from looking at the first non-trivial Picard iterate to (11) that one starts to loose regularity as soon as the initial data is rougher than $H^{1+\frac{1}{4}}$ (see [6]). Furthermore, by an adaptation of the $(3+1)$ dimensional counterexamples of Lindblad [ $[$, one should be able to show that certain instances of the equations (11) are ill-posed in the Sobolev spaces $H^{s}$ when $s<1+\frac{1}{4}$. This is in stark contrast to the situation in $(5+1)$ and higher dimensions, where one can come arbitrarily close to the scale invariant Sobolev space $H^{\frac{n-2}{2}}$ 14, and can in fact recover local existence in the scale invariant Besov space $\dot{B}^{\frac{n-2}{2}, 1}$ in $(6+1)$ and higher dimensions [11].

The reason why the low dimensional setting is more difficult to control than the higher dimensional regime it that "parallel" interactions in the non-linearity on the right hand side of (11) become stronger and stronger as the dimension decreases. Closely related to this is the range of validity of the so called Strichartz estimates. Specifically, in $(4+1)$ dimensions, one looses the $L^{2}\left(L^{4}\right)$ Strichartz estimate which clearly plays a major role via Duhamel's principle in the well posedness theory equations with quadratic type interactions (that is, one looks to put the non-linearity in $L^{1}\left(L^{2}\right)$ ). For an important class of equations with special structure in the nonlinearity, this interaction of parallel waves is largely destroyed, and one can gain the needed improvement over the $H^{1+\frac{1}{4}}$ barrier to come arbitrarily close to the scaling. For example, this was accomplished by Klainerman-Tataru in [7] for the Yang-Mills equations (4) with the Coulomb gauge enforced. Going even further in this direction, it should be possible to combine the Besov space technique of [11] with the compound null structure ${ }^{3}$ discussed in [9] to push the global well-posedness theory of these (Coulomb gauge) equations to the scale invariant Besov space $\dot{B}^{1,1}$. Finally it is conjectured, and a major open problem of this subject, that by either working with the curvature (2) directly or by making use of the Coulomb gauge restriction of the equations (4), the equations (3) are well posed in the scale invariant Sobolev space $\dot{H}^{1}$.

\footnotetext{
${ }^{3}$ A close inspection of the proof in 7 will show that this is needed to get around the failure of certain end-point bilinear $L^{1}\left(L^{\infty}\right)$ estimates. Specifically, compounding the non-linearity of (4) in the Coulomb gauge, and taking into account various cancellations due to the null structures present, one arrives at a set of equations that morally looks like $\square \phi=\Delta^{-1}(\phi \nabla \phi) \cdot \nabla \phi$. This equation looks a lot like wave-maps, except that the weights are distributed in a more unfavorable fashion. In particular, while it is true that one can get $\Delta^{-1}(\phi \nabla \phi) \hookrightarrow L^{1}\left(L^{\infty}\right)$ at fixed frequency, there is no room left to add over the low frequencies in a High $\times$ High $\Rightarrow$ Low interaction. Therefore, even if one assumes a Besov structure for the $\phi$, there is not enough room to close. However, this is exactly the bad frequency interaction which is eliminated by the tri-linear null structure of 9 .
} 
However, our interest here is in the Lorentz gauge equations (6), and more generally equations which are generically of the type (11). An inspection of the nonlinearity in (6) reveals that it does not seem to contain the special "null structure" of the non-linearity of (4) in the Coulomb gauge (at least at the bilinear level), and it is a tentative conjecture that these specific equations are in fact ill-posed for regularities less than $H^{1+\frac{1}{4}}$. This brings into question whether one can prove scale invariant global existence in the spirit of [11. It is clear from the above discussion that any modification to that theory will need to go away from translation invariant spaces. That is, one is led to look for a theory which includes the low regularity micro-local techniques of [11, but somehow makes crucial use of the weighted vector-field from [3. One idea is to understand how the presence of homogeneous weighted derivatives effects the range of validity of the Strichartz estimates. Because the main obstacle to improved estimates of this type is the presence of waves which are highly concentrated along a given null direction, it is natural to expect that the rotation generators:

$$
\Omega_{i j}=x_{i} \partial_{j}-x_{j} \partial_{i},
$$

play a distinguished role because they penalize such objects. This indeed turns out to be the case, and one gains a significant improvement at the level of both linear and bilinear estimates as was discussed in [12. This observation will form the basis for the first main ingredient of the approach we take here, which is to better control the linear theory. At the non-linear level, one would expect that the rotations (14) also play a major role because they would help to eliminate parallel interactions coming in the right hand side of (11). In other words, one would hope that in some sense the rotations (14) could substitute for the null-structures one makes use of in the Coulomb gauge. Again, this turns out to be the case and will form the second main pillar of the approach we take here which is to build function spaces that take into account "angular concentration" phenomena. What we will do is prove prove the following theorem:

Theorem 1.1 (Global well posedness for the system (11)). For the generic system of non-linear wave equations (11) on $(4+1)$ dimensional Minkowski space, there exists constants $0<\epsilon_{0}, C$ such that if

$$
\left\|\left(f^{I}, g^{I}\right)\right\|_{\dot{B}_{\Omega}^{1,1} \times \dot{B}_{\Omega}^{0,1}} \leqslant \epsilon_{0},
$$

where $\dot{B}_{\Omega}^{1,1}$ is the Banach space with norm:

$$
\|h\|_{\dot{B}_{\Omega}^{1,1}}=\|h\|_{\dot{B}^{1,1}}+\sum_{i<j}\left\|\Omega_{i j} h\right\|_{\dot{B}^{1,1}},
$$

and likewise for $B_{\Omega}^{0,1}$, then there exits a global solution $\psi^{I}$ to the system (11) with initial data $\left(f^{I}, g^{I}\right)$ which satisfies the stability condition:

$$
\left\|\psi^{I}\right\|_{C\left(\dot{B}_{\Omega}^{1,1}\right) \cap C^{(1)}\left(\dot{B}_{\Omega}^{0,1}\right)} \leqslant C\left\|\left(f^{I}, g^{I}\right)\right\|_{\dot{B}_{\Omega}^{1,1} \times \dot{B}_{\Omega}^{0,1}} .
$$

In particular, there is no energy growth of the solution to (11). The solution $\psi^{I}$ is unique and depends smoothly on the initial data in the following sense: There exists a sequence of smooth functions $\left(f_{N}^{I}, g_{N}^{I}\right)$ such that:

$$
\lim _{N \rightarrow \infty}\left\|\left(f^{I}, g^{I}\right)-\left(f_{N}^{I}, g_{N}^{I}\right)\right\|_{\dot{B}_{\Omega}^{1,1} \times \dot{B}_{\Omega}^{0,1}}=0 .
$$


For this sequence of functions, there exists a sequence of unique smooth global solutions $\psi_{N}^{I}$ of (11) with this initial data. Furthermore, the $\psi_{N}^{I}$ converge to $\psi^{I}$ as follows:

$$
\lim _{N \rightarrow \infty}\left\|\psi^{I}-\psi_{N}^{I}\right\|_{C\left(\dot{B}_{\Omega}^{1,1}\right) \cap C^{(1)}\left(\dot{B}_{\Omega}^{0,1}\right)}=0 .
$$

Also, $\psi^{I}$ is the only solution which may be obtained as a limit (in the above sense) of solutions to (11) with regularizations of $\left(f^{I}, g^{I}\right)$ as initial data. Finally, $\psi^{I}$ retains any extra smoothness inherent in the initial data. That is, if $\left(f^{I}, g^{I}\right)$ also has finite $\dot{H}_{\Omega}^{s} \times \dot{H}_{\Omega}^{s-1}$ norm, for $1<s$, then so does $\psi^{I}$ at fixed time and one has the following estimate:

$$
\left\|\psi^{I}\right\|_{C\left(\dot{H}_{\Omega}^{s}\right) \cap C^{(1)}\left(\dot{H}_{\Omega}^{s-1}\right)} \leqslant C\left\|\left(f^{I}, g^{I}\right)\right\|_{\dot{H}_{\Omega}^{s} \times \dot{H}_{\Omega}^{s-1}} .
$$

In a straightforward way, our estimates also address the issue of the asymptotic freedom of the system (11). As an immediate corollary of our approach, we have that:

Theorem 1.2. Using the same notation as above, for our solution $\psi^{I}$ to the system (11) with initial data $\left(f^{I}, g^{I}\right)$, there exists data sets $\left(f^{I^{ \pm}}, g^{I^{ \pm}}\right)$, such that if $\psi^{I^{ \pm}}$is the solution to the homogeneous wave equation, $\square \psi^{I^{ \pm}}=0$, with this initial data, the following asymptotics hold:

$$
\begin{aligned}
\lim _{t \rightarrow \infty}\left\|\psi^{I^{+}}-\psi^{I}\right\|_{\dot{B}_{\Omega}^{1,1} \cap \partial_{t} \dot{B}_{\Omega}^{0,1}} & =0, \\
\lim _{t \rightarrow-\infty}\left\|\psi^{I^{-}}-\psi^{I}\right\|_{\dot{B}_{\Omega}^{1,1} \cap \partial_{t} \dot{B}_{\Omega}^{0,1}} & =0 .
\end{aligned}
$$

Furthermore, the scattering operator retains any additional regularity inherent in the initial data. That is, if $\left(f^{I}, g^{I}\right)$ has finite $\dot{H}_{\Omega}^{s} \times \dot{H}_{\Omega}^{s-1}$ norm, then so does $\left(f^{I^{ \pm}}, g^{I^{ \pm}}\right)$, and the following asymptotics hold:

$$
\begin{aligned}
\lim _{t \rightarrow \infty}\left\|\psi^{I^{+}}-\psi^{I}\right\|_{\dot{H}_{\Omega}^{s} \cap \partial_{t} \dot{H}_{\Omega}^{s-1}} & =0, \\
\lim _{t \rightarrow-\infty}\left\|\psi^{I^{-}}-\psi^{I}\right\|_{\dot{H}_{\Omega}^{s} \cap \partial_{t} \dot{H}_{\Omega}^{s-1}} & =0 .
\end{aligned}
$$

Remark 1.3. In the statement of the generic system (11) and in proof of Theorem 1.1 we have ignored the cubic type interactions $\left(\phi^{I}\right)^{3}$ which appear on the left hand side of (6). Notice that these terms respect the scaling (12). It turns out that they are trivial to treat in the spaces we use here by taking a product of the $L^{3}\left(L^{6}\right)$ Strichartz estimate which is available in $(4+1)$ dimension. The only real issue is to make sure that one recovers the Besov structure for $\mathrm{High} \times \mathrm{High} \Rightarrow$ Low frequency interactions, but this is again a triviality due to the room in the $L^{3}\left(L^{6}\right)$ estimate (one does not even have to use bilinear estimates to do this).

Remark 1.4. For convenience, we have chosen to work here with the spaces (16) which involve a whole angular (momentum) derivative. As the reader will see shortly, there is much room in the dyadic estimates of our proof. Specifically, it should be possible to prove our theorem with the use of only a little more then $\frac{1}{2}$ an angular derivative. However, this would force one to work out $L^{\infty}$ paraproducts 
in the angular variable, which would bring another layer of technical complications that we have chosen to avoid. However, this still leaves an interesting gap because based on the local theory one would expect that, say for compactly supported initial data, there is global regularity for small $H^{1+\frac{1}{4}+\epsilon}$ norm. Therefore, in some sense, our estimates seem to fall $\frac{1}{4}$ a derivative short of the optimal level. Perhaps this gap can be eliminated by somehow incorporating (fractional powers of) the other invariant vector-fields. In particular, the boosts $\Omega_{0 i}=t \partial_{i}+x_{i} \partial_{t}$. We will say no more of this here.

Remark 1.5. We have not included here a specific discussion of the first set of model equations (9). It turns out that these are a bit easier to treat than the equations (11). In other words, the difference between (9) and (11) which can be seen at the level of decay can also be seen at the micro-local level. Specifically, for the equations (9), the estimate (114) below would be much easier to prove because it would not need the bilinear estimates (35). It should be noted however, that the somewhat more involved version of the estimate (114) which we use here can also directly be used in the proof of the well-posedness of equations of type (9).

\section{Notation AND PRELIMINARY SETUP}

For quantities $A$ and $B$, we denote by $A \lesssim B$ to mean that $A \leqslant C \cdot B$ for some large constant $C$. The constant $C$ may change from line to line, but will always remain fixed for any given instance where this notation appears. Likewise we use the notation $A \sim B$ to mean that $\frac{1}{C} \cdot B \leqslant A \leqslant C \cdot B$. We also use the notation $A \ll B$ to mean that $A \leqslant \frac{1}{C} \cdot B$ for some large constant $C$. This is the notation we will use throughout the paper to break down quantities into the standard cases: $A \sim B$, or $A \ll B$, or $B \ll A$; and $A \lesssim B$, or $B \ll A$, without ever discussing which constants we are using. We will also employ the following notation to indicate arbitrarily small adjustments to a given numerical value: For a given constant $A$, we write $A+($ resp. $A-)$ to mean that for any sufficiently small $0<\epsilon$, we may replace $A+$ by $A+\epsilon$ (resp. $A-\epsilon$ ) on the line where it occurs and still have a true estimate. However, we do not assume any uniformity in this notation. That is, any implicit constants which appear in conjunction with $A \pm$ may depend on $\epsilon$. An example of this is the $L^{\infty}$ Sobolev estimate:

$$
\|f\|_{L^{\infty}\left(\mathbb{R}^{4}\right)} \lesssim\|f\|_{H^{2+}\left(\mathbb{R}^{4}\right)} .
$$

Also, if two separate occurrences of the $A \pm$ notation appear on the same line, we will not assume that the same $\epsilon$ is being used for each separate occurrence.

For a given function of two variables $(t, x) \in \mathbf{R} \times \mathbf{R}^{\mathbf{4}}$ we write the spatial and space-time Fourier transform as:

$$
\begin{aligned}
\widehat{u}(t, \xi) & =\int e^{-2 \pi i \xi \cdot x} u(t, x) d x \\
\widetilde{u}(\tau, \xi) & =\int e^{-2 \pi i(\tau t+\xi \cdot x)} u(t, x) d t d x
\end{aligned}
$$


respectively. Because we are not assuming any extra structure in the non-linearity of (11), we will work almost exclusively with the space-time Fourier transform.

For a given function $f$ of the spatial variable only, we denote by:

$$
\begin{aligned}
e^{i t \sqrt{-\Delta}} f(x) & =\int e^{\pi i(t|\xi|+\xi \cdot x)} \hat{f}(\xi) d \xi, \\
e^{-i t \sqrt{-\Delta}} f(x) & =\int e^{\pi i(-t|\xi|+\xi \cdot x)} \hat{f}(\xi) d \xi,
\end{aligned}
$$

the forward and backward wave propagation of $f$.

Let $E$ denote any fundamental solution to the homogeneous wave equation: i.e., one has the formula $\square E=\delta$. We define the standard Cauchy parametrix for the wave equation via the rule:

$$
\frac{1}{\square} F=E * F-W(E * F) .
$$

Here and in the sequel, for any test function $H$ we use the notation $W(H)$ to denote the solution to the homogeneous wave equation with initial data $\left(H(0), \partial_{t} H(0)\right)$. Explicitly, one has the identity:

$$
\widehat{\frac{1}{\square} F}(t, \xi)=-\int_{0}^{t} \frac{\sin (2 \pi|\xi|(t-s))}{2 \pi|\xi|} \widehat{F}(s, \xi) d s .
$$

For any function $F$ which is supported away from the light cone in Fourier space, we shall use the following notation for division by the symbol of the wave equation:

$$
\frac{1}{\Xi} F=E * F
$$

Of course, the definition of $\frac{1}{\Xi}$ does not depend on $E$ so long as for $F$ is supported away from the light cone; and for us that will always be the case when we use this notation. Explicitly, one has the formula:

$$
\mathcal{F}\left[\frac{1}{\Xi} F\right](\tau, \xi)=\frac{1}{4 \pi^{2}\left(\tau^{2}-|\xi|^{2}\right)} \widetilde{F}(\tau, \xi) .
$$

Next, we record here some basic results from spherical harmonic analysis. For more details on this material, see the companion paper to this work [12]. The first order of business concerns defining fractional powers of the spherical Laplacean:

$$
\Delta_{s p h}=\sum_{i<j} \Omega_{i j}^{2}
$$

As is well known, this can be done via spectral resolution, and we write:

$$
|\Omega|^{s}=\left(-\Delta_{\text {sph }}\right)^{\frac{s}{2}} \text {. }
$$

The operator (24) kills off the spherically symmetric part of any function it is applied to. Because of this, we will employ the following "inhomogeneous" version of this operator:

$$
\langle\Omega\rangle^{s} f=F_{0}+|\Omega|^{s} f,
$$


where $f$ is a function of the spatial variable, and $f_{0}$ denotes the spherically symmetric part of $f$. That is:

$$
f_{0}(r)=\frac{1}{\left|\mathbb{S}^{3}\right|} \int_{\mathbb{S}^{3}} f(r \omega) d \omega .
$$

A key property of the operators $\langle\Omega\rangle^{s}$ is that they commute with the spatial (and thus space-time) Fourier transform:

$$
\widehat{\langle\Omega\rangle^{s f}}=\langle\Omega\rangle^{s} \widehat{f} .
$$

Also, we have the following equivalence of Sobolev type norms ${ }^{4}$ involving the unit power $\langle\Omega\rangle$ :

$$
\|f\|_{H_{\Omega}^{1}\left(\mathbb{R}^{4}\right)}^{2}=\|\langle\Omega\rangle f\|_{L^{2}\left(\mathbb{R}^{4}\right)}^{2}=\left\|f_{0}\right\|_{L^{2}\left(\mathbb{R}^{4}\right)}^{2}+\sum_{i<j}\left\|\Omega_{i j} f\right\|_{L^{2}\left(\mathbb{R}^{4}\right)}^{2} .
$$

Because all of the norms we build here will be based on the unit powers $\langle\Omega\rangle$, we will by abuse of notation replace any instance of a single $\Omega_{i j}$ with the operator $\langle\Omega\rangle$, and although it is not strictly true, we will assume that there is the point-wise Leibniz rule:

$$
\langle\Omega\rangle(f g)=\langle\Omega\rangle f \cdot g+f \cdot\langle\Omega\rangle g .
$$

This will be a great convenience to us because some of the norms we define below are $L^{\infty}$ based (in particular (63) and (68)), where it would be difficult to define paraproducts for fractional powers $\langle\Omega\rangle^{s}$.

Finally, we record here two basic results which follow from the Littlewood-Paley theorem for the sphere, in conjunction with interpolation in weighted spaces of the type $L^{p}\left(\ell_{s}^{2}\right)$ (again, see [12] for details):

Proposition 2.1 (Sobolev embedding on the sphere). If $F$ is a test function on the unit sphere $\mathbb{S}^{3} \subset \mathbb{R}^{4}$, then the following estimate holds for $2 \leqslant p<\infty$ :

$$
\|F\|_{L^{p}\left(\mathbb{S}^{3}\right)} \lesssim\left\|\langle\Omega\rangle^{3\left(\frac{1}{2}-\frac{1}{p}\right)} F\right\|_{L^{2}\left(\mathbb{S}^{3}\right)},
$$

where the implicit constants depend only on $p$. In particular, if $f$ is a test function on $\mathbb{R}^{4}$ and $s<\frac{3}{2}$, then one has:

$$
\int_{0}^{\infty}\|f(r)\|_{L^{\frac{6}{3-2 s}\left(\mathbb{S}^{3}\right)}}^{2} r^{3} d r \lesssim \int_{0}^{\infty}\left\|\langle\Omega\rangle^{s} f(r)\right\|_{L^{2}\left(\mathbb{S}^{3}\right)}^{2} r^{3} d r=\|f\|_{H_{\Omega}^{s}\left(\mathbb{R}^{4}\right)}
$$

Proposition 2.2 (Interpolation of spherical Sobolev spaces). Let $W_{\Omega}^{s, p}$ denote the norm:

$$
\|f\|_{W_{\Omega}^{s, p}\left(\mathbb{R}^{4}\right)}=\left\|\langle\Omega\rangle^{s} f\right\|_{L^{p}\left(\mathbb{R}^{4}\right)}
$$

for functions $f$ of the spatial variable only. Then for $1<p_{1}, p_{2}<\infty$ one has the following interpolation spaces:

$$
\left(W_{\Omega}^{s_{1}, p_{1}}, W_{\Omega}^{s_{2}, p_{2}}\right)_{t}=W_{\Omega}^{s, p},
$$

where $s=(1-t) s_{1}+t s_{2}$ and $\frac{1}{p}=\frac{(1-t)}{p_{1}}+\frac{t}{p_{2}}$.

\footnotetext{
${ }^{4}$ The $\Omega$ subscript in conjunction with numerical superscripts, e.g. the $s$ in $H_{\Omega}^{s}$, will always denote angular derivatives in this section. In other places in the paper the $\Omega$ subscript will always mean one angular derivative, while the superscripts will denote translation invariant derivatives. An example of this is the notation $\dot{B}_{\Omega}^{1,1}$ introduced on line 16.
} 


\section{Strichartz Estimates}

We list here the space-time estimates for the homogeneous wave equations which form the foundation for our proof of Theorem 1.1 As we have mentioned before, all of these are of "Strichartz type". The first group of estimates we will use are just the classical Strichartz estimates for the wave equation which we state for the case of $(4+1)$ dimensions:

Proposition 3.1 (Frequency localized "classical" Strichartz estimates on $\mathbb{R}^{(4+1)}$ including endpoints (see [2])). Let $n=4$ be the number of spatial dimensions, and let $\sigma=\frac{3}{2}$ be the corresponding Strichartz admissible exponent. If $f$ is any function of the spatial variable only, denote by $f_{1}=P_{1} f$ its unit frequency projection (see (38)). Then one has the following family estimates for $2 \leqslant q$ :

$$
\left\|e^{i t \sqrt{-\Delta}} f_{1}\right\|_{L^{q}\left(L^{r}\right)} \lesssim\left\|f_{1}\right\|_{L^{2}}
$$

where $\frac{1}{q}+\frac{\sigma}{r} \leqslant \frac{\sigma}{2}$.

As was discussed in the introduction, the estimates (28) alone are not strong enough to close a global iteration argument for non-linear wave equations of the form (11). What is needed is an improvement of the range of admissible $(q, r)$ indices on the left hand side of (28). It is well known that this cannot be accomplished within the context of translation invariant smoothness assumptions on the initial data (see [12]). However, incorporating extra weighted smoothness assumptions for the angular variable provides the needed mechanism to overcome this obstacle. The corresponding estimates are:

Proposition 3.2 (Frequency localized Strichartz estimates for angularly regular initial data (see [12])). Let $n=4$ be the number of spatial dimensions, and let $\sigma_{\Omega}=3$ denote the four dimensional angular Strichartz admissible exponent. Let $f_{1}$ be a unit frequency function of the spacial variable only (as above). Then for indices $(q, r)$ such that $\frac{1}{q}+\frac{\sigma}{r} \geqslant \frac{\sigma}{2}$ and $\frac{1}{q}+\frac{\sigma_{\Omega}}{r}<\frac{\sigma_{\Omega}}{2}$, and for every $0<\epsilon$, there is a $C_{\epsilon}$ which depends only on $\epsilon$ such that the following estimates hold:

$$
\left\|e^{i t \sqrt{-\Delta}} f_{1}\right\|_{L^{q}\left(L^{r}\right)} \lesssim C_{\epsilon}\left\|\langle\Omega\rangle^{s} f_{1}\right\|_{L^{2}}
$$

where $s=(1+\epsilon)\left(\frac{n-1}{r}+\frac{2}{q}-\frac{n-1}{2}\right)$.

In practice, only a small subset of the indices $(q, r)$ in the two propositions listed above will be of use to us. These are $(\infty, 2),(2, \infty),(2,6)$, and $(2,3+)$. To highlight this fact, we list out the corresponding instances of (28) and (29): 


$$
\begin{aligned}
\left\|e^{i t \sqrt{-\Delta}} f_{1}\right\|_{L^{\infty}\left(L^{2}\right)} & \lesssim\left\|f_{1}\right\|_{L^{2}} \\
\left\|e^{i t \sqrt{-\Delta}} f_{1}\right\|_{L^{2}\left(L^{\infty}\right)} & \lesssim\left\|f_{1}\right\|_{L^{2}}, \\
\left\|e^{i t \sqrt{-\Delta}} f_{1}\right\|_{L^{2}\left(L^{6}\right)} & \lesssim\left\|f_{1}\right\|_{L^{2}} \\
\left\|\langle\Omega\rangle^{\frac{1}{2}} e^{i t \sqrt{-\Delta}} f_{1}\right\|_{L^{2}\left(L^{3+}\right)} & \lesssim \|\left\langle\Omega f_{1} \|_{L^{2}}\right.
\end{aligned}
$$

In our proof of Theorem 1.1] we will need more than just the linear estimates (28) - (29). This is a common feature of lower dimensional problems, and is necessitated by the presence of certain bad High $\times \mathrm{High} \Rightarrow$ Low frequency interactions. The standard device for dealing with this problem is the use of bilinear Strichartz estimates. We will use here T. Tao's fine-course scale idea for dealing with these (see [5] and [13]). The basic idea is to fix a scale, say $\frac{1}{\mu}$ for $\mu \ll 1$, and then decompose the domain of the spatial variable into cubes with side lengths $\sim \frac{1}{\mu}$. Then, one replaces the usual $L^{r}$ norm in the spatial variable with $\ell^{r}\left(L^{2}\right)$, where the $L^{2}$ norm is taken on the "fine" scale of each individual cube, while the $\ell^{r}$ norm represents the "coarse" scale which is summation over all cubes. One reason this method is so powerful, is that it allows one to use the bilinear construction process directly in an iteration procedure, where resorting to the canned estimates that this method ultimately provides may be unduly burdensome. This is crucial when dealing with eccentric multipliers as we do here. Therefore, we will only state the two scale estimates themselves, without mentioning the various bilinear estimates which follow as a corollary. For point of reference, we point out here that these estimates will only be used in the proof of estimate (114) below. We begin by stating the classical two-scale estimates:

Proposition 3.3 (Frequency localized two-scale Strichartz estimates ([5] , [13])). Let $n=4$ be the number of spatial dimensions. Let $0<\mu \lesssim 1$ be a given parameter. Let $\left\{Q_{\alpha}\right\}$ be a partition of $\mathbb{R}^{n}$ into cubes of side length $\sim \frac{1}{\mu}$. Then if $f_{1}$ is a unit frequency function of the spatial variable only, the following estimates hold:

$$
\left\|\left(\sum_{\alpha}\left\|e^{i t \sqrt{-\Delta}} f_{1}\right\|_{L^{2}\left(Q_{\alpha}\right)}^{r}\right)^{\frac{1}{r}}\right\|_{L_{t}^{2}} \lesssim \mu^{-1}\left\|f_{1}\right\|_{L^{2}},
$$

where $6 \leqslant r$.

Next, we state the improvement to (34) which incorporates angular regularity:

Proposition 3.4 (Frequency localized two-scale Strichartz estimates for angularly regular data; endpoint case (12)). Let $n=4$ be the number of spatial dimensions and let $f_{1}$ be a unit frequency function of the spatial variable only. Let $0<\mu \lesssim 1$ be given, and let $\left\{Q_{\alpha}\right\}$ be a partition of $\mathbb{R}^{n}$ into cubes of side length $\sim \frac{1}{\mu}$. Then for 
every $0<\epsilon$, there is a $C_{\epsilon}$ and $3<r_{\epsilon}$ depending on $\epsilon$, such that $r_{\epsilon} \rightarrow 3$ as $\epsilon \rightarrow 0$ such that the following estimate holds:

$$
\left\|\left(\sum_{\alpha}\left\|e^{i t \sqrt{-\Delta}} f_{1}\right\|_{L^{2}\left(Q_{\alpha}\right)}^{r_{\epsilon}}\right)^{\frac{1}{r_{\epsilon}}}\right\|_{L_{t}^{2}} \lesssim C_{\epsilon} \mu^{-\frac{1}{2}-2 \epsilon}\left\|\langle\Omega\rangle^{\frac{1}{2}+\epsilon} f_{1}\right\|_{L^{2}} .
$$

In practice, we will only need a single instance of both (34) and (35). We state these in encapsulated form here for the convenience of the reader:

$$
\begin{gathered}
\left\|\left(\sum_{\alpha}\left\|e^{i t \sqrt{-\Delta}} f_{1}\right\|_{L^{2}\left(Q_{\alpha}\right)}^{6}\right)^{\frac{1}{6}}\right\|_{L_{t}^{2}} \lesssim \mu^{-1}\left\|f_{1}\right\|_{L^{2}}, \\
\left\|\left(\sum_{\alpha}\left\|e^{i t \sqrt{-\Delta}} f_{1}\right\|_{L^{2}\left(Q_{\alpha}\right)}^{3+}\right)^{\frac{1}{3+}}\right\|_{L_{t}^{2}} \lesssim \mu^{-\left(\frac{1}{2}+\right)}\left\|\langle\Omega\rangle f_{1}\right\|_{L^{2}} .
\end{gathered}
$$

Notice that we have added an extra $\frac{1}{2}$ an angular derivative to the right hand side of (37) above. This is how we will use this estimate in this paper, and is a reflection of the fact that we have elected to work with integral powers of the angular momentum operator $|\Omega|$ in this work.

\section{Multipliers, Functions Spaces, and Scattering}

In this section, we will set up much of notation to be used in the proof of Theorem 1.1 and we will construct the function spaces used to iterate the problem (11). For the most part, the approach taken here is similar to that of [11, with the simple addition of angular derivatives. A notable exception occurs in the definition of the special $L^{1}\left(L^{\infty}\right)$ "outer block" norms (66) and (68). Because of the need to capture extra savings in our estimates based on angular regularity, these are a bit more involved than their cousins used in 11]. We strongly recommend that the reader first read that paper as a warm up to the present work because it represents a simplified version of the type of decompositions and estimate combinations used here.

4.1. Multipliers and angular restrictions. Let $\varphi$ be a smooth bump function (i.e. supported on the set $|s| \leqslant 2$ such that $\varphi=1$ for $|s| \leqslant 1$ ). In what follows, it will be a great convenience for us to assume that $\varphi$ may change its exact form for two separate instances of the symbol $\varphi$ (even if they occur on the same line). In this way, we may assume without loss of generality that in addition to being smooth, we also have the idempotence identity $\varphi^{2}=\varphi$. We shall use this convention for all the cutoff functions we introduce in the sequel. 
For $\lambda \in 2^{\mathbb{Z}}$, we denote the dyadic scaling of $\varphi$ by $\varphi_{\lambda}(s)=\varphi\left(\frac{s}{\lambda}\right)$. The most basic Fourier localizations we shall use here are with respect to the spatial and space-time Fourier variable and the distance from the cone in Fourier space. Accordingly, we form the Littlewood-Paley type cutoff functions:

$$
\begin{aligned}
p_{\lambda}(\xi) & =\varphi_{2 \lambda}(|\xi|)-\varphi_{\frac{1}{2} \lambda}(|\xi|), \\
s_{\lambda}(\tau, \xi) & =\varphi_{2 \lambda}(|(\tau, \xi)|)-\varphi_{\frac{1}{2} \lambda}(|(\tau, \xi)|), \\
c_{d}(\tau, \xi) & =\varphi_{2 d}(|\tau|-|\xi|)-\varphi_{\frac{1}{2} d}(|\tau|-|\xi|) .
\end{aligned}
$$

We now denote the corresponding Fourier multiplier operator via the formulas $\widetilde{S_{\lambda} u}=s_{\lambda} \widetilde{u}$ and $\widetilde{C_{d} u}=c_{d} \widetilde{u}$ respectively. We also use a multi-subscript notation to denote products of the above operators, e.g. $S_{\lambda, d}=S_{\lambda} C_{d}$. We shall use the notation:

$$
S_{\lambda, \bullet<}=\sum_{\delta \leqslant d} S_{\lambda, \delta}
$$

to denote cutoff in an $O(d)$ neighborhood of the light cone in Fourier space. At times it will also be convenient to write $S_{\lambda, d \leqslant \bullet}=S_{\lambda}-S_{\lambda, \bullet<d}$. We shall also use the notation $S_{\lambda, d}^{ \pm}$etc. to denote the multiplier $S_{\lambda, d}$ cutoff in the half space $\pm \tau>0$.

The other type of Fourier localization which will be central to our analysis will be the restriction of the spatial angular variable $\omega$, where $\xi=|\xi| \omega$. We accomplish this as follows: For each small parameter $\eta \lesssim 1$, we decompose the unit sphere in $\mathbb{R}^{4}$ into angular sectors of size $\sim \eta$ with bounded overlap independent of $\eta$. We label the corresponding partition of unity by their angles and write them as $b_{\eta}^{\omega}$. It is clear that this construction can be done in such a way that all the $b_{\eta}^{\omega}$ are (essentially) rotations of each other. Note that the multipliers $b_{\eta}^{\omega} p_{\lambda}$ essentially cutoff on parallelepipeds of size $\lambda \times(\lambda \eta) \times(\lambda \eta) \times(\lambda \eta)$. Furthermore, after rotating each of these multipliers onto the $\xi_{1}$ axis, one has the following bounds:

$$
\left|\partial_{1}^{N} b_{\eta}^{\omega} p_{\lambda}\right| \leqslant C_{N} \lambda^{-N}, \quad \quad\left|\partial_{i}^{N} b_{\eta}^{\omega} p_{\lambda}\right| \leqslant C_{N}(\lambda \eta)^{-N},
$$

for $i=2,3,4$. In particular we see that each operator $B_{\eta}^{\omega} P_{\lambda}$ is given by convolution with an $L^{1}$ kernel,

A major defect of the $S_{\lambda, d}$ multipliers is that they are not uniformly bounded on most Lebesgue spaces. However, if we first localize them further onto a block that is directed along the light cone in Fourier space of dimensions $\lambda \times \sqrt{\lambda d} \times \sqrt{\lambda d} \times \sqrt{\lambda d} \times d$, then the resulting kernels will be uniformly in $L^{1}$. In the sequel we shall write these special block localizations as:

$$
S_{\lambda, d}^{\omega}=B_{\left(\frac{d}{\lambda}\right)^{\frac{1}{2}}}^{\omega} P_{\lambda} S_{\lambda, d}, \quad S_{\lambda, \bullet \leqslant d}^{\omega}=B_{\left(\frac{d}{\lambda}\right)^{\frac{1}{2}}}^{\omega} P_{\lambda} S_{\lambda, \bullet \leqslant d} .
$$

It is important to note that the above multipliers are cutoffs in the region of Fourier space where $|\tau| \lesssim|\xi|$. We now record some useful multiplier bounds, the proofs of which can be found in [11]:

Lemma 4.1 (Multiplier boundedness on Lebesgue spaces). 
(1) The following multipliers are given by $L^{1}$ kernels: $\quad \lambda^{-1} \nabla S_{\lambda}, B_{\left(\frac{d}{\lambda}\right)^{\frac{1}{2}}}^{\omega} P_{\lambda}$, $S_{\lambda, d}^{\omega}$, and $(\lambda d) \Xi^{-1} S_{\lambda, d}^{\omega}$. In particular, all of these are bounded on every mixed Lebesgue space $L^{q}\left(L^{r}\right)$.

(2) The following multipliers are bounded on the spaces $L^{q}\left(L^{2}\right)$, for $1 \leqslant q \leqslant \infty$ : $S_{\lambda, d}$, and $S_{\lambda, \bullet \leqslant d}$.

In the sequel, we shall also need the following somewhat stronger version of the boundedness of the multipliers $S_{\lambda, d}^{\omega}$ and $(\lambda d) \Xi^{-1} S_{\lambda, d}^{\omega}$ :

Lemma 4.2 (Multiplier boundedness on special $L^{1}\left(L^{\infty}\right)$ spaces). Let $u$ be a function of space and time, then the following estimates hold:

$$
\begin{aligned}
\int \sup _{\omega}\left\|\frac{1}{\Xi} S_{\lambda, d}^{\omega} u(t)\right\|_{L^{\infty}} d t & \lesssim \frac{1}{\lambda d} \int \sup _{\omega}\left\|S_{\lambda, d}^{\omega} u(t)\right\|_{L^{\infty}} d t \\
\int\left(\sum_{\omega}\left\|\frac{1}{\Xi} S_{\lambda, d}^{\omega} u(t)\right\|_{L^{\infty}}^{2}\right)^{\frac{1}{2}} d t & \lesssim \frac{1}{\lambda d} \int\left(\sum_{\omega}\left\|S_{\lambda, d}^{\omega} u(t)\right\|_{L^{\infty}}^{2}\right)^{\frac{1}{2}} d t \\
\int \sup _{\omega}\left\|S_{\lambda, d}^{\omega} u(t)\right\|_{L^{\infty}} d t & \lesssim \int \sup _{\omega}\left\|B_{\left(\frac{d}{\lambda}\right)^{\frac{1}{2}}}^{\omega} P_{\lambda} S_{\lambda} u(t)\right\|_{L^{\infty}} d t \\
\int\left(\sum_{\omega}\left\|S_{\lambda, d}^{\omega} u(t)\right\|_{L^{\infty}}^{2}\right)^{\frac{1}{2}} d t & \lesssim \int\left(\sum_{\omega}\left\|B_{\left(\frac{d}{\lambda}\right)^{\frac{1}{2}}}^{\omega} P_{\lambda} S_{\lambda} u(t)\right\|_{L^{\infty}}^{2}\right)^{\frac{1}{2}} d t
\end{aligned}
$$

proof of estimates (43)-(46). It suffices to prove the implications (43) and (44), as the proofs of (45) and (46) follow from virtually identical reasoning. Using the idempotence relation $S_{\lambda, d}^{\omega}=S_{\lambda, d}^{\omega} S_{\lambda, d}^{\omega}$, and writing $K^{\omega}$ for the convolution kernel of the operator $(\lambda d) \Xi^{-1} S_{\lambda, d}^{\omega}$, for the estimate (43) we can bound:

$$
\begin{aligned}
& (\lambda d) \int \sup _{\omega}\left\|\frac{1}{\Xi} S_{\lambda, d}^{\omega} u(t)\right\|_{L_{x}^{\infty}} d t \\
= & \int \sup _{\omega}\left\|K^{\omega} * S_{\lambda, d}^{\omega} u(t)\right\|_{L_{x}^{\infty}} d t, \\
\lesssim & \int\left(\sup _{\omega} \int\left\|K^{\omega}(t-s)\right\|_{L_{x}^{1}}\left\|S_{\lambda, d}^{\omega} u(s)\right\|_{L_{x}^{\infty}} d s\right) d t, \\
\lesssim & \iint \sup _{\omega}\left\|K^{\omega}(t-s)\right\|_{L_{x}^{1}} \sup _{\omega}\left\|S_{\lambda, d}^{\omega} u(s)\right\|_{L_{x}^{\infty}} d s d t .
\end{aligned}
$$

We now use the fact that all of the $K^{\omega}$ are essentially spatial rotations of each other to show that for each fixed $(t-s)$ :

$$
\left\|K^{\omega}(t-s)\right\|_{L_{x}^{1}} \lesssim\left\|K^{\omega_{0}}(t-s)\right\|_{L_{x}^{1}}
$$


where $\omega_{0}$ is, say, the angular sector in the direction of the $\xi_{1}$ axis in Fourier space. Thus, we have that:

$$
\begin{aligned}
& (\lambda d) \int \sup _{\omega}\left\|\frac{1}{\Xi} S_{\lambda, d}^{\omega} u(t)\right\|_{L_{x}^{\infty}} d t \\
\lesssim & \iint\left\|K^{\omega_{0}}(t-s)\right\|_{L_{x}^{1}} \sup _{\omega}\left\|S_{\lambda, d}^{\omega} u(s)\right\|_{L_{x}^{\infty}} d s d t . \\
\lesssim & \left\|K^{\omega_{0}}\right\|_{L^{1}\left(L^{1}\right)} \int \sup _{\omega}\left\|S_{\lambda, d}^{\omega} u(s)\right\|_{L_{x}^{\infty}} d s . \\
\lesssim & \int \sup _{\omega}\left\|S_{\lambda, d}^{\omega} u(s)\right\|_{L_{x}^{\infty}} d s .
\end{aligned}
$$

Similarly, for the estimate (44), we compute:

$$
\begin{aligned}
& (\lambda d) \int\left(\sum_{\omega}\left\|\frac{1}{\Xi} S_{\lambda, d}^{\omega} u(t)\right\|_{L_{x}^{\infty}}^{2}\right)^{\frac{1}{2}} d t \\
= & \int\left(\sum_{\omega}\left\|K^{\omega} * S_{\lambda, d}^{\omega} u(t)\right\|_{L_{x}^{\infty}}^{2}\right)^{\frac{1}{2}} d t \\
\lesssim & \int\left(\sum_{\omega}\left(\int\left\|K^{\omega}(t-s)\right\|_{L_{x}^{1}}\left\|S_{\lambda, d}^{\omega} u(s)\right\|_{L_{x}^{\infty}} d s\right)^{2}\right)^{\frac{1}{2}} d t \\
\lesssim & \iint\left(\sum_{\omega}\left\|K^{\omega}(t-s)\right\|_{L_{x}^{1}}^{2}\left\|S_{\lambda, d}^{\omega} u(s)\right\|_{L_{x}^{\infty}}^{2}\right)^{\frac{1}{2}} d s d t . \\
\lesssim & \iint \sup _{\omega}\left\|K^{\omega}(t-s)\right\|_{L_{x}^{1}}\left(\sum_{\omega}\left\|S_{\lambda, d}^{\omega} u(s)\right\|_{L_{x}^{\infty}}^{2}\right)^{\frac{1}{2}} d s d t . \\
\lesssim & \left\|K^{\omega_{0}}\right\|_{L^{1}\left(L^{1}\right)} \int\left(\sum_{\omega}\left\|S_{\lambda, d}^{\omega} u(s)\right\|_{L_{x}^{\infty}}^{2}\right)^{\frac{1}{2}} d s . \\
\lesssim & \int\left(\sum_{\omega}\left\|S_{\lambda, d}^{\omega} u(s)\right\|_{L_{x}^{\infty}}^{2}\right)^{\frac{1}{2}} d s .
\end{aligned}
$$

This completes the proof of estimates (43)-(46).

4.2. Function spaces. Our next step will be to use the above multipliers to define dyadic versions of the function spaces we will Picard iterate in. We first define those dyadic norms which guarantee that the various Strichartz estimates of Theorems 3.13 .23 .3 and 3.4 hold. These are:

$$
\begin{array}{rlr}
\|u\|_{X_{\lambda, p}^{\frac{1}{2}}}^{p} & =\sum_{d \in 2^{\mathbb{Z}}} d^{\frac{p}{2}}\left\|S_{\lambda, d} u\right\|_{L^{2}}^{p}, & \text { ("classical" } H^{s, \delta} \text { ) } \\
\|u\|_{Y_{\lambda}} & =\lambda^{-1}\left\|\square S_{\lambda} u\right\|_{L^{1}\left(L^{2}\right)} . & \text { (Duhamel) }
\end{array}
$$

Because these function spaces give no weight to solutions to the homogeneous wave equation, we will always need to use them in concert with the fixed frequency energy 
space $S_{\lambda}\left(L^{\infty}\left(L^{2}\right)\right)$. Grouping all of these together, we form our first main dyadic function space:

$$
\|u\|_{F_{\lambda}}=\left(X_{\lambda, 1}^{\frac{1}{2}}+Y_{\lambda}\right) \cap S_{\lambda}\left(L^{\infty}\left(L^{2}\right)\right) .
$$

We also define the corresponding norms with angular derivatives added as follows:

$$
\|u\|_{F_{\Omega, \lambda}}=\|\langle\Omega\rangle u\|_{F_{\lambda}} .
$$

At times it will also be convenient for us to write:

$$
\begin{aligned}
\|u\|_{X_{\Omega, \lambda, 1}^{\frac{1}{2}}} & =\|\langle\Omega\rangle u\|_{X_{\lambda, 1}^{\frac{1}{2}}}, \\
\|u\|_{Y_{\Omega, \lambda}} & =\|\langle\Omega\rangle u\|_{Y_{\lambda} .} .
\end{aligned}
$$

A key property of the spaces $F_{\lambda}$ and $F_{\Omega, \lambda}$ is that their elements can be written as integrals over solutions to the homogeneous wave equation where the integration involves only the temporal variable (see [11] for details). This is sometimes referred to as foliation, or the trace method. Using this technique, the Strichartz estimates from Theorems 3.1 3.2 3.3 and 3.4 can be transferred to the $F$ spaces in a straightforward way, even though some of the estimates involve weighted angular derivatives. This is one of the main reasons why the use of Strichartz estimates which only involve the rotation vector fields is so crucial to the approach taken in this paper. Indeed, if the Strichartz estimates (29) and (35) contained any of the other invariant vector fields from [3], there would be no way to transfer them to the $F_{\Omega, \lambda}$ via usual temporal foliation. We now list the various foliated instances of estimates (28), (29), (34), and (35) which will be used in this paper:

$$
\begin{aligned}
\left\|S_{1} u\right\|_{L^{\infty}\left(L^{2}\right)} & \lesssim\|u\|_{F_{1}}, \\
\left\|S_{1} u\right\|_{L^{2}\left(L^{\infty}\right)} & \lesssim\|u\|_{F_{1}}, \\
\left\|S_{1} u\right\|_{L^{2}\left(L^{6}\right)} & \lesssim\|u\|_{F_{1}}, \\
\left\|S_{1}\langle\Omega\rangle^{\frac{1}{2}} u\right\|_{L^{2}\left(L^{3+}\right)} & \lesssim\|u\|_{F_{\Omega, 1}}, \\
\left\|S_{\lambda} u\right\|_{L^{\infty}\left(L^{2}\right)} & \lesssim\|u\|_{F_{\lambda}}, \\
\left\|S_{\lambda} u\right\|_{L^{2}\left(L^{6}\right)} & \lesssim \lambda^{\frac{5}{6}}\|u\|_{F_{\lambda}}, \\
\left\|S_{\lambda}\langle\Omega\rangle^{\frac{1}{2}} u\right\|_{L^{2}\left(L^{3+}\right)} & \lesssim \lambda^{\frac{1}{6}+}\|u\|_{F_{\Omega, \lambda}}, \\
\left\|\left(\sum_{\alpha}\left\|S_{1} u(t)\right\|_{L^{2}\left(Q_{\alpha}\right)}^{6}\right)^{\frac{1}{6}}\right\|_{L_{t}^{2}} & \lesssim \mu^{-1}\|u\|_{F_{1}}, \\
\left\|\left(\sum_{\alpha}\left\|S_{1} u(t)\right\|_{L^{2}\left(Q_{\alpha}\right)}^{3+}\right)^{\frac{1}{3+}}\right\|_{L_{t}^{2}} & \lesssim \mu^{-\left(\frac{1}{2}+\right)}\|u\|_{F_{\Omega, 1}} .
\end{aligned}
$$

An important property of the $Y_{\lambda}$ spaces is that they are nearly contained in the $X_{\lambda, 1}^{\frac{1}{2}}$ spaces. More precisely, this is true at a fixed dyadic distance from the light 
cone in Fourier space. To see this, note that by duality and the estimate (51), we have the inclusions:

$$
\lambda \Xi^{-1} L^{1}\left(L^{2}\right) \subseteq \lambda \Xi^{-1} X_{\lambda, \infty}^{-\frac{1}{2}} \subseteq X_{\lambda, \infty}^{\frac{1}{2}} .
$$

Using the above embedding at fixed distance from the cone, and by dyadic summing, shows that we have the following estimates:

$$
\begin{aligned}
d^{\frac{1}{2}}\left\|S_{\lambda, d} u\right\|_{L^{2}\left(L^{2}\right)} & \lesssim\|u\|_{F_{\lambda}} \\
d^{\frac{1}{2}}\left\|S_{\lambda, d \leqslant \bullet} u\right\|_{L^{2}\left(L^{2}\right)} & \lesssim\|u\|_{F_{\lambda}} .
\end{aligned}
$$

It is well known, the norms (47) and (48) are not strong enough to iterate wave equations which contain derivatives in their nonlinearities like (11). This is due to the presence of a very specific Low $\times H i g h$ frequency interaction in the term $\phi \nabla \phi$. What is needed to circumvent this problem is to add some extra $L^{1}\left(L^{\infty}\right)$ norms to the $F$ spaces. This idea originally goes back to the work of Klainerman-Machedon [4, and was later used to its full extent in Tataru [14. In our previous work [11, we used a slight innovation on the norms in 14] which allowed one to work in a scale invariant setting. Here it will be necessary for us to use somewhat more technical versions of those norms, in part because some of the estimates we need to prove here are essentially tri-linear in nature. To define these norms for a given test function $u$ at frequency $\lambda$, we first consider all ways that one may write:

$$
u=\sum_{\alpha} u^{\alpha} .
$$

For each $u^{\alpha}$, we consider a set of solid angles, $\left\{\theta_{\alpha, d}\right\}$, with the property that each $\sqrt{\frac{d}{\lambda}} \leqslant\left|\theta_{\alpha, d}\right|$. We then measure:

$$
\left\|u^{\alpha}\right\|_{Z_{\lambda}^{\left\{\theta_{\alpha, d}\right\}}}=\sum_{d}\left|\theta_{\alpha, d}\right| \int\left(\sum_{\theta_{\alpha, d}} \sup _{\omega \subseteq \theta_{\alpha, d}}\left\|S_{\lambda, d}^{\omega} u^{\alpha}(t)\right\|_{L_{x}^{\infty}}^{2}\right)^{\frac{1}{2}} d t .
$$

In the above expression, the inclusion $\omega \subseteq \theta_{\alpha, d}$ indicates that the solid angle $\omega$, when considered as a spherical cap of dimension $\sqrt{\frac{d}{\lambda}} \times \sqrt{\frac{d}{\lambda}} \times \sqrt{\frac{d}{\lambda}}$ (i.e. one has $\left.|\omega| \lesssim \sqrt{\frac{d}{\lambda}}\right)$, is contained in the spherical cap defined by $\theta_{\alpha, d}$. It is important to note that the multipliers $S_{\lambda, d}^{\omega}$ and $(\lambda d) \Xi^{-1} S_{\lambda, d}^{\omega}$ are bounded on the norm (63). The proof of this is similar to the proofs of (44) and (46) above. We record these facts as: 
(64)

$$
\int\left(\sum_{\theta} \sup _{\omega \subseteq \theta}\left\|\frac{1}{\Xi} S_{\lambda, d}^{\omega} u(t)\right\|_{L_{x}^{\infty}}^{2}\right)^{\frac{1}{2}} d t \lesssim \frac{1}{\lambda d} \int\left(\sum_{\theta} \sup _{\omega \subseteq \theta}\left\|S_{\lambda, d}^{\omega} u(t)\right\|_{L_{x}^{\infty}}^{2}\right)^{\frac{1}{2}} d t
$$

$$
\int\left(\sum_{\theta} \sup _{\omega \subseteq \theta}\left\|S_{\lambda, d}^{\omega} u(t)\right\|_{L_{x}^{\infty}}^{2}\right)^{\frac{1}{2}} d t \lesssim \int\left(\sum_{\theta} \sup _{\omega \subseteq \theta}\left\|B_{\left(\frac{d}{\lambda}\right)^{\frac{1}{2}}}^{\omega} P_{\lambda} u(t)\right\|_{L_{x}^{\infty}}^{2}\right)^{\frac{1}{2}} d t .
$$

We now define the $Z_{\lambda}$ norms to be the infimum over all possible choices of the sum (62) and the angle sets $\left\{\theta_{\alpha, d}\right\}$ :

$$
\|u\|_{Z_{\lambda}}=\inf _{u=\sum_{\alpha} u^{\alpha}}\left(\inf _{\left\{\theta_{\alpha, d}\right\}} \sum_{\alpha}\left\|u^{\alpha}\right\|_{Z_{\lambda}^{\left\{\theta_{\alpha, d}\right\}}}\right) .
$$

It is important to note here that in the vast majority of instances, we will only need to estimate (63) for $u^{\alpha}=u$ and $\left|\theta_{\alpha, d}\right| \sim \sqrt{\frac{d}{\lambda}}$. That is, for the most part we will be dealing with the norm:

$$
\|u\|_{Z_{\lambda}^{\prime}}=\sum_{d}\left(\frac{d}{\lambda}\right)^{\frac{1}{2}} \int\left(\sum_{\omega}\left\|S_{\lambda, d}^{\omega} u(t)\right\|_{L_{x}^{\infty}}^{2}\right)^{\frac{1}{2}} d t .
$$

Notice that one has the inclusion $Z_{\lambda}^{\prime} \subseteq Z_{\lambda}$, so it is sufficient to be able to bound the right hand side of (67). The only instance where it is more convenient to work with the larger angles in the norm (66), is in estimate (126) below. See the comments in the proof of that estimate for more information as to why (66) is necessary.

We will also need an analog of the $Z_{\lambda}$ norm for situations where $u$ cannot absorb an extra angular derivative. This is given by the following:

$$
\|u\|_{Z_{\Omega, \lambda}}=\sum_{d} \int \sup _{\omega}\left\|S_{\lambda, d}^{\omega} u(t)\right\|_{L_{x}^{\infty}} d t .
$$

The reason we have defined $Z_{\Omega, \lambda}$ separately instead of defining it as $Z_{\lambda}$ is mainly for notational convenience, as it will give a uniform look to the statements of various estimates which follow. We now add the norms (66) and (68) together with the norm (50) to form the fixed frequency version of the main function space which we will iterate in:

$$
G_{\Omega, \lambda}=F_{\Omega, \lambda} \cap \lambda|\Omega|^{-1} Z_{\lambda} \cap \lambda Z_{\Omega, \lambda},
$$

where $\lambda|\Omega|^{-1} Z_{\lambda}$ is the space with the norm $\lambda^{-1}\||\Omega| u\|_{Z_{\lambda}}$, while $\lambda Z_{\Omega, \lambda}$ has the norm $\lambda^{-1}\|u\|_{Z_{\Omega, \lambda}}$. The overall spaces we will use are the analogs of the Besov 
space $\dot{B}^{1,1}$ and the Sobolev spaces $\dot{H}^{s}$ when $1<s$ :

$$
\begin{aligned}
\|u\|_{G_{\Omega}^{1}} & =\sum_{\lambda} \lambda\|u\|_{G_{\Omega, \lambda}}, \\
\|u\|_{F_{\Omega}^{s}}^{2} & =\sum_{\lambda} \lambda^{2 s}\|u\|_{F_{\Omega, \lambda}}^{2} .
\end{aligned}
$$

The space $G_{\Omega}^{1}$ will be the foundation of our iteration procedure, while the $F_{\Omega}^{s}$ space is axillary and will be used to show that solutions to (11) retain any extra regularity inherent in the initial data.

4.3. Scattering in the $F$ spaces. As it turns out, our scattering result, Theorem 1.2 is contained for free in the structure of the $F$ spaces in the sense that any element of those spaces can be approximated by a free wave (solution to the homogeneous wave equation) at temporal infinity. Using a straightforward approximation argument where one truncates the very high and very low frequencies, we can reduce things to proving scattering for functions truncated at a fixed dyadic frequency:

Lemma 4.3 (Scattering in the space $F_{\Omega, \lambda}$ ). For any function $u_{\lambda} \in F_{\Omega, \lambda}$, there exists a set of initial data $\left(f_{\lambda}^{ \pm}, g_{\lambda}^{ \pm}\right) \in P_{\lambda}\left(L_{\Omega}^{2}\right) \times \lambda P_{\lambda}\left(L_{\Omega}^{2}\right)$ such that the following asymptotic holds:

$$
\begin{aligned}
\lim _{t \rightarrow \infty}\left\|u_{\lambda}(t)-W\left(f_{\lambda}^{+}, g_{\lambda}^{+}\right)(t)\right\|_{\dot{H}_{\Omega}^{1} \cap \partial_{t}\left(L_{\Omega}^{2}\right)} & =0 \\
\lim _{t \rightarrow-\infty}\left\|u_{\lambda}(t)-W\left(f_{\lambda}^{-}, g_{\lambda}^{-}\right)(t)\right\|_{\dot{H}_{\Omega}^{1} \cap \partial_{t}\left(L_{\Omega}^{2}\right)} & =0 .
\end{aligned}
$$

proof of Lemma 4.3. The proof depends on the fact that one may write:

$$
u_{\lambda}=u_{\dot{X}_{\Omega, \lambda}}+u_{X_{\Omega, \lambda, 1}^{1 / 2}}^{+}+u_{X_{\Omega, \lambda, 1}^{1 / 2}}^{-}+u_{Y_{\Omega, \lambda}}
$$

where $u_{\dot{X}_{\Omega, \lambda}}$ is a solution to the homogeneous wave equation with $L_{\Omega}^{2}$ data, $u_{X_{\Omega, \lambda, 1}^{1 / 2}}^{ \pm}$ are functions in $X_{\Omega, \lambda, 1}^{1 / 2}$ whose Fourier transforms are also functions and are cut off in the upper (resp. lower) half plane (in Fourier space), and $u_{Y_{\Omega, \lambda}}$ is in the space $Y_{\Omega, \lambda}$. For a discussion of this, see [1]. We now define the scattering data implicitly by the relations:

$$
\begin{aligned}
& W\left(f_{\lambda}^{+}, g_{\lambda}^{+}\right)(t)=u_{\dot{X}_{\Omega, \lambda}}+\int_{0}^{\infty}\left|D_{x}\right|^{-1} \sin \left(\left|D_{x}\right|(t-s)\right) \square u_{Y_{\Omega, \lambda}}(s) d s, \\
& W\left(f_{\lambda}^{-}, g_{\lambda}^{-}\right)(t)=u_{\dot{X}_{\Omega, \lambda}}+\int_{-\infty}^{0}\left|D_{x}\right|^{-1} \sin \left(\left|D_{x}\right|(t-s)\right) \square u_{Y_{\Omega, \lambda}}(s) d s .
\end{aligned}
$$

Using the fact that $\square u_{Y_{\Omega, \lambda}}$ has finite $L^{1}\left(L_{\Omega}^{2}\right)$ norm, we are reduced to showing the limits:

$$
\lim _{t \rightarrow \pm \infty}\left\|u_{X_{\Omega, \lambda, 1}^{1 / 2}}^{+}(t)+u_{X_{\Omega, \lambda, 1}^{1 / 2}}^{-}(t)\right\|_{\dot{H}_{\Omega}{ }^{1} \cap \partial_{t}\left(L_{\Omega}^{2}\right)}=0 .
$$

This is a straightforward exercise in Plancherel's theorem and Dominated Convergence for sequences of integrals. The key is to use the foliation of the $X_{\Omega, \lambda, 1}^{1 / 2}$ 
spaces alluded to above and the fact that all weighted derivatives involve the spatial variable only. We refer the interested reader to the work [1] for a full account.

\section{Some Preliminary Estimates of Sobolev Type}

In this section we provide some basic estimates of Sobolev type which will be needed in the sequel, as well as some inclusions which result from these for the function spaces we introduced in the last section. We begin with the basic local Sobolev estimate, also know as Bernstein's inequality:

Lemma 5.1 (Local Sobolev estimates). Let $u$ be a test function on $\mathbb{R}^{4}$, then one has the following frequency localized estimates:

$$
\left\|B_{\eta}^{\omega} P_{1} u\right\|_{L^{p}} \lesssim \eta^{3\left(\frac{1}{r}-\frac{1}{p}\right)}\|u\|_{L^{r}}
$$

For the convenience of the reader, we highlight here some specific instances of (174), some of which have been rescaled, that will be used in the sequel. In all of the estimates below, the integration is taken over $\mathbb{R}^{4}$ :

$$
\begin{aligned}
\left\|B_{d^{\frac{1}{2}}}^{\omega} P_{1} u\right\|_{L^{\infty}} & \lesssim d^{\frac{1}{4}}\|u\|_{L^{6}}, \\
\left\|B_{\left(\frac{d}{\mu}\right)^{\frac{1}{2}}}^{\omega} P_{\mu} u\right\|_{L^{\infty}} & \lesssim \mu^{\frac{5}{12}} d^{\frac{1}{4}}\|u\|_{L^{6}}, \\
\left\|B_{d^{\frac{1}{2}}}^{\omega} P_{1} u\right\|_{L^{\infty}} & \lesssim d^{\frac{1}{2}-}\|u\|_{L^{3+}}, \\
\left\|B_{\left(\frac{d}{\mu}\right)^{\frac{1}{2}}}^{\omega} P_{\mu} u\right\|_{L^{\infty}} & \lesssim \mu^{\frac{5}{6}-} d^{\frac{1}{2}-}\|u\|_{L^{3+}}, \\
\left\|B_{\left(\frac{d}{\mu}\right)^{\frac{1}{2}}}^{\omega} P_{\mu} u\right\|_{L^{\infty}} & \lesssim \mu^{\frac{5}{4}-} d^{\frac{3}{4}-}\|u\|_{L^{2+}}, \\
\left\|B_{\left(\frac{d}{\mu}\right)^{\frac{1}{2}}}^{\omega} P_{\mu} u\right\|_{L^{\infty}} & \lesssim \mu^{\frac{5}{4}} d^{\frac{3}{4}}\|u\|_{L^{2}}, \\
\left\|B_{\left(\frac{d}{\mu}\right)^{\frac{1}{2}}}^{\omega} P_{\mu} u\right\|_{L^{\infty}} & \lesssim \mu^{\frac{5}{3}-} d^{1-}\|u\|_{L^{\frac{3}{2}+}}, \\
\left\|B_{\left(\frac{d}{\mu}\right)^{\frac{1}{2}}}^{\omega} P_{\mu} u\right\|_{L^{2}} & \lesssim \mu^{\frac{5}{12}} d^{\frac{1}{4}}\|u\|_{L^{\frac{3}{2}}}, \\
\left\|B_{\left(\frac{d}{\mu}\right)^{\frac{1}{2}}}^{\omega} P_{\mu} u\right\|_{L^{2}} & \lesssim \mu^{\frac{5}{6}-} d^{\frac{1}{2}-}\|u\|_{L^{\frac{6}{5}+}}, \\
\left\|P_{\mu} u\right\|_{L^{2}} & \lesssim \mu^{2}\|u\|_{L^{1}} .
\end{aligned}
$$

It will be of crucial importance to us in the resolution of Theorem 1.1 to know that our solutions to (11) are not concentrating on small angular regions in Fourier space. This control of the solutions we construct will allow us to use the improved 
Strichartz estimates (29) and (35). However, because we must test our solutions for angular regularity to gain this information, it will be necessary for us to deal with situations where we need to squeeze some extra savings out of a term that has no extra translation invariant derivatives to give and therefore cannot be put in any other space than $L^{\infty}\left(L^{2}\right)$. In other words, situations where there is extra angular regularity present in the absence of dispersion. An example of this is a Low $\times$ High frequency interaction where the angular derivative falls on the low frequency term. In some sense, this is the major technical difficulty which needs to be overcome when applying the estimates (29) and (35) to nonlinear problems which contain derivatives in the nonlinearity. To deal with this problem, we shall employ the following Lemma which we state for arbitrary spatial dimension. This is essentially an uncertainty principle for how a function can be localized in the angular variable in Fourier space:

Lemma 5.2 (Angular concentration estimates). Let $2 \leqslant n$ be a given integer. Then for any test function $u$ on $\mathbb{R}^{n}$, and any $2 \leqslant p<\infty$ one has the following estimate:

$$
\sup _{\omega}\left\|B_{\eta}^{\omega} u\right\|_{L^{p}} \lesssim \eta^{s}\left\|\langle\Omega\rangle^{s} u\right\|_{L^{p}}
$$

where $0 \leqslant s<\frac{n-1}{p}$.

proof of estimate 850. Upon rotation onto the positive $\xi$ axis in Fourier space, the multiplier $b_{\eta}^{\omega}(\xi)$ satisfies the following differential bounds:

$$
\left|\partial_{\xi_{i_{1}}} \ldots \partial_{\xi_{i_{k}}} b_{\eta}^{\omega}(\xi)\right| \lesssim 2^{-j k}, \quad \xi_{1} \in\left[2^{j}, 2^{j+1}\right] .
$$

Furthermore, for each fixed fixed $\xi_{1} \in\left[2^{j}, 2^{j+1}\right]$, one has that the support of $b_{\eta}^{\omega}\left(\xi_{1}, \xi^{\prime}\right)$ lies in (perhaps some thickening of) the region $\xi^{\prime} \in\left[-2^{j+1}, 2^{j+1}\right] \times \ldots \times$ $\left[-2^{j+1}, 2^{j+1}\right]$. Therefore, for every permutation of the variables $\left(\xi_{1}, \ldots, \xi_{n}\right)$, one has the following integral bound:

$$
\sup _{0<k \leqslant n} \sup _{\xi_{k+1}, \ldots, \xi_{n}} \int_{\mathcal{D}}\left|\partial_{\xi_{1}} \ldots \partial_{\xi_{k}} b_{\eta}^{\omega}\left(\xi_{1}, \ldots, \xi_{k} ; \xi_{k+1}, \ldots, \xi_{n}\right)\right| d \xi_{1} \ldots d \xi_{k} \lesssim 1
$$

where $\mathcal{D}$ is any dyadic rectangle ${ }^{5}$ on $\mathbb{R}^{k}$. Therefore, by the Marcinkiewicz Multiplier Theorem (see for instance p. 109 of [10]), one has that for $2 \leqslant p<\infty$ :

$$
\left\|B_{\eta}^{\omega} u\right\|_{L^{p}} \lesssim\|u\|_{L^{p}}
$$

Therefore, by using the $n$-dimensional version of Proposition 2.2 it suffices to show the following estimate in $L^{2}$ :

$$
\left\|B_{\eta}^{\omega} u\right\|_{L^{2}} \lesssim \eta^{s}\left\|\langle\Omega\rangle^{s} u\right\|_{L^{2}}, \quad s<\frac{n-1}{2} .
$$

By the Plancherel Theorem and the fact that $\langle\Omega\rangle^{s}$ commutes with the Fourier transform, this is equivalent to showing that:

$$
\left\|b_{\eta}^{\omega} \widehat{u}\right\|_{L^{2}} \lesssim \eta^{s}\left\|\langle\Omega\rangle^{s} \widehat{u}\right\|_{L^{2}}, \quad s<\frac{n-1}{2} .
$$

\footnotetext{
${ }^{5}$ That is, one generated by the usual dyadic partition of the coordinate axis.
} 
This can now be accomplished via a simple use of Hölders inequality followed by the $n$ dimensional version of the angular Sobolev embedding Proposition (26) as follows: For $s<\frac{n-1}{2}$ we compute that:

$$
\begin{aligned}
\left\|b_{\eta}^{\omega} \widehat{u}\right\|_{L^{2}}^{2} & =\int_{0}^{\infty}\left\|b_{\eta}^{\omega} \widehat{u}(r)\right\|_{L^{2}\left(\mathbb{S}^{n-1}\right)}^{2} r^{n-1} d r \\
& \lesssim \int_{0}^{\infty}\left\|b_{\eta}^{\omega}\right\|_{L^{\frac{n-1}{s}}\left(\mathbb{S}^{n-1}\right)}^{2}\|\widehat{u}(r)\|_{L^{\frac{2(n-1)}{n-2 s}\left(\mathbb{S}^{n-1}\right)}}^{2} r^{n-1} d r \\
& \lesssim \eta^{2 s} \int_{0}^{\infty}\left\|\langle\Omega\rangle^{s} \widehat{u}(r)\right\|_{L^{2}\left(\mathbb{S}^{n-1}\right)}^{2} r^{n-1} d r \\
& =\eta^{2 s}\left\|\langle\Omega\rangle^{s} \widehat{u}\right\|_{L^{2}}^{2} .
\end{aligned}
$$

This ends the proof (85).

We list here some specific incarnations of 85ich will appear in the sequel. These norms are taken over $\mathbb{R}^{4}$ for the Lebesgue spaces, and $\mathbb{R}^{(4+1)}$ for the $F$ spaces:

$$
\begin{aligned}
& \sup _{\omega}\left\|B_{\left(\frac{d}{\mu}\right)^{\frac{1}{2}}}^{\omega} u\right\|_{L^{3+}} \lesssim\left(\frac{d}{\mu}\right)^{\frac{1}{4}}\left\|\langle\Omega\rangle^{\frac{1}{2}} u\right\|_{L^{3+}}, \\
& \sup _{\omega}\left\|B_{\left(\frac{d}{\mu}\right)^{\frac{1}{2}}}^{\omega} u\right\|_{L^{2}} \lesssim\left(\frac{d}{\mu}\right)^{\frac{1}{2}}\|\langle\Omega\rangle u\|_{L^{2}} \\
& \sup _{\omega}\left\|B_{\left(\frac{d}{\mu}\right)^{\frac{1}{2}}}^{\omega} u\right\|_{F_{\lambda}} \lesssim\left(\frac{d}{\mu}\right)^{\frac{1}{2}}\|u\|_{F_{\Omega, \lambda}}
\end{aligned}
$$

We conclude this section by using the Lemmas $5.2[5.1$ to show that the $Z$ norms need only be recovered for those pieces of $u \in F_{\Omega, \lambda}$ which are in the $X$ space portion of things:

Lemma 5.3 ( $Z$ norm recovery for functions in the $Y$ spaces). On $\mathbb{R}^{4+1}$ one has the following uniform inclusions:

$$
\begin{aligned}
Y_{\lambda} & \subseteq \lambda Z_{\lambda}^{\prime}, \\
Y_{\Omega, \lambda} & \subseteq \lambda Z_{\Omega, \lambda} .
\end{aligned}
$$


proof of the inclusions (89) and (90). We'll start with the inclusion (89). For a fixed $d$, we can use the special multiplier bound (44) to compute that:

$$
\begin{aligned}
& \int\left(\sum_{\omega}\left\|S_{\lambda, d}^{\omega} u(t)\right\|_{L_{x}^{\infty}}^{2}\right)^{\frac{1}{2}} d t, \\
\lesssim & \int\left(\sum_{\omega}\left\|\frac{1}{\Xi} S_{\lambda, d}^{\omega} \square u(t)\right\|_{L_{x}^{\infty}}^{2}\right)^{\frac{1}{2}} d t, \\
\lesssim & \frac{1}{\lambda d} \int\left(\sum_{\omega}\left\|S_{\lambda, d}^{\omega} \square u(t)\right\|_{L_{x}^{\infty}}^{2}\right)^{\frac{1}{2}} d t .
\end{aligned}
$$

We now use the local Sobolev estimate (80) and the multiplier boundedness Lemma 4.1 to conclude that:

$$
\begin{aligned}
& \int\left(\sum_{\omega}\left\|S_{\lambda, d}^{\omega} \square u(t)\right\|_{L_{x}^{\infty}}^{2}\right)^{\frac{1}{2}} d t, \\
& \lesssim \lambda^{\frac{5}{4}} d^{\frac{3}{4}} \int\left(\sum_{\omega}\left\|S_{\lambda, d}^{\omega} \square u(t)\right\|_{L_{x}^{2}}^{2}\right)^{\frac{1}{2}} d t, \\
& \lesssim \lambda^{\frac{5}{4}} d^{\frac{3}{4}} \int\left\|S_{\lambda, d} \square u(t)\right\|_{L_{x}^{2}} d t, \\
& \lesssim \lambda^{\frac{5}{4}} d^{\frac{3}{4}}\left\|\square S_{\lambda} u\right\|_{L^{1}\left(L^{2}\right)} .
\end{aligned}
$$

Multiplying through by the factor $(\lambda d)^{-1}(d / \lambda)^{\frac{1}{2}}$ summing the last line of the above estimate over $d$ yields:

$$
\begin{aligned}
\|u\|_{Z_{\lambda}^{\prime}} & \lesssim \sum_{d}\left(\frac{d}{\lambda}\right)^{\frac{1}{4}}\left\|\square S_{\lambda} u\right\|_{L^{1}\left(L^{2}\right)} \\
& \lesssim \lambda\|u\|_{Y_{\lambda}}
\end{aligned}
$$

To prove the inclusion (90), we first use the special multiplier bound (43), the local Sobolev estimate (80), and the concentration estimate [87] to compute that for fixed $d$ one has:

$$
\begin{aligned}
& \int \sup _{\omega}\left\|S_{\lambda, d}^{\omega} u(t)\right\|_{L_{x}^{\infty}} d t, \\
\lesssim & \frac{1}{\lambda d} \int \sup _{\omega}\left\|S_{\lambda, d}^{\omega} \square u(t)\right\|_{L_{x}^{\infty}} d t, \\
\lesssim & \left(\frac{\lambda}{d}\right)^{\frac{1}{4}} \int \sup _{\omega}\left\|S_{\lambda, d}^{\omega} \square u(t)\right\|_{L_{x}^{2}} d t, \\
\lesssim & \left(\frac{d}{\lambda}\right)^{\frac{1}{4}} \int\left\|S_{\lambda, d} \square\langle\Omega\rangle u(t)\right\|_{L_{x}^{2}} d t .
\end{aligned}
$$


Using the multiplier bound (4.1) and summing over $d$ now yields:

$$
\begin{aligned}
\|u\|_{Z_{\Omega, \lambda}} & \lesssim \sum_{d}\left(\frac{d}{\lambda}\right)^{\frac{1}{4}}\left\|S_{\lambda, d}\langle\Omega\rangle u\right\|_{L^{1}\left(L^{2}\right)}, \\
& \lesssim \lambda\|u\|_{Y_{\Omega, \lambda}} .
\end{aligned}
$$

\section{Bilinear Decompositions for Small Angles}

In this section, we will list various bilinear decompositions for frequency localized products of the form:

$$
S_{\mu}\left(S_{\lambda_{1}} u \cdot S_{\lambda_{2}} v\right) .
$$

The discussion here will be in the same style as Section 8 of [11, and we will leave some of the details to that paper. Our first task will be to decompose certain instances of the product (91), where $\lambda_{1} \sim \lambda_{2}$ and $\mu \lesssim \lambda_{1}$. This, of course, will be used in the High $\times$ High type frequency interactions in the sequel. Here we will pay special attention to the localized product:

$$
S_{\mu, d}\left(S_{\lambda, \bullet \leqslant \min \{d, c \mu\}} u \cdot S_{\lambda, \bullet \leqslant \min \{d, c \mu\}} v\right),
$$

where $c \ll 1$ is some small constant which we shall fixed in the proof. The most important feature of (92) is that the two terms in the product are restricted to be closer to the light-cone in Fourier space than their output. By a simple computation of the convolution variables, this in turn implies that the angle of interaction between these two terms is restricted to within $O\left(\frac{d}{\mu}\right)$ of the angle of the output (in Fourier space of course). To see this, notice that all we are talking about here is a matter of the support of the cutoff function associated with the various multipliers in (92). Therefore, it suffices to study the convolution:

$$
s_{\mu, d}\left(s_{\lambda, \bullet \leqslant \min \{d, c \mu\}} * s_{\lambda, \bullet \leqslant \min \{d, c \mu\}}\right),
$$

Because of the restrictions involved, we can assume without loss of generality that the two terms in the convolution (93) are supported in the lower resp. upper half

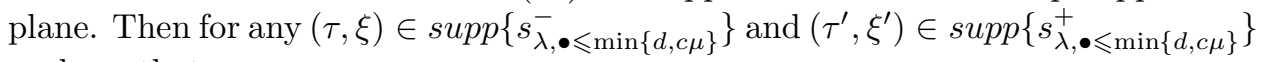
we have that:

$$
\begin{aligned}
O(d) & =|| \tau+\tau^{\prime}|-| \xi+\xi^{\prime}||, \\
& =|||\xi|-\left|\xi^{\prime}\right|+O(d)|-| \xi+\xi^{\prime}||, \\
& =|O(d)+||\xi|-\left|\xi^{\prime}\right||-| \xi+\xi^{\prime}|| .
\end{aligned}
$$

Therefore we are able to conclude that:

$$
\lambda \Theta_{\xi,-\xi^{\prime}}^{2} \lesssim|||\xi|-\left|\xi^{\prime}\right||-| \xi+\xi^{\prime}||=O(d)
$$

showing that we in fact have $\left|\Theta_{\xi,-\xi^{\prime}}\right| \lesssim \sqrt{\frac{d}{\lambda}}$. However, this extra precision will not concern us here, and it will suffice to know that $\left|\Theta_{\xi,-\xi^{\prime}}\right| \lesssim \sqrt{\frac{d}{\mu}}$. It will also be important for us to know that the angle between $\xi+\xi^{\prime}$ and $\pm \xi$ does not exceed 
$O\left(\sqrt{\frac{d}{\mu}}\right)$. This information will be used to decompose products of the form (93) by only localizing one of the factors in Fourier space. This will be crucial to us in the proof of estimate (114) below, where the use of eccentric multipliers in conjunction with the coarse scale decomposition of physical space needed for Proposition 3.4 will not be possible due to the uncertainty principle. To obtain this type of decomposition, we compute:

$$
\begin{aligned}
O(d) & =|| \tau^{\prime}|-| \xi^{\prime}||, \\
& =||\left(\tau+\tau^{\prime}\right)-\tau|-|\left(\xi+\xi^{\prime}\right)-\xi||, \\
& =|| \pm\left|\xi+\xi^{\prime}\right|-|\xi|+O(d)|-|\left(\xi+\xi^{\prime}\right)-\xi||, \\
& =|O(d)+| \pm\left|\xi+\xi^{\prime}\right|-|\xi|-\left|\left(\xi+\xi^{\prime}\right)-\xi\right| \mid, \\
& =\left|O(d)+O\left(\mu \Theta_{\xi+\xi^{\prime}, \pm \xi}^{2}\right)\right| .
\end{aligned}
$$

Therefore we must have that $O\left(\mu \Theta_{\xi+\xi^{\prime}, \pm \xi}^{2}\right)=O(d)$, or $\Theta_{\xi+\xi^{\prime}, \pm \xi}=O\left(\sqrt{\frac{d}{\mu}}\right)$.

The other piece of information we will need to know here is that the restricted convolution (93) is supported in the region where $\left|\tau+\tau^{\prime}\right| \lesssim\left|\xi+\xi^{\prime}\right|$. This will be used to deconstruct the $s_{\mu, d}$ multiplier that appears there into a sum over the angular pieces $s_{\mu, d}^{\omega}$, each of which is supported in that region. We calculate:

$$
\begin{aligned}
\left|\tau+\tau^{\prime}\right| & =|| \xi|-| \xi^{\prime}|+O(\min \{c \mu, d\})|, \\
& \lesssim|| \xi|-| \xi^{\prime}||+O(\min \{c \mu, d\}), \\
& \lesssim\left|\xi+\xi^{\prime}\right|+O(\min \{c \mu, d\}) .
\end{aligned}
$$

A quick look at the support of $s_{\mu, d}$ shows that if one had $\left|\xi+\xi^{\prime}\right| \ll\left|\tau-\tau^{\prime}\right|$, one must have $\left|\xi+\xi^{\prime}\right| \ll \mu$ and $\left|\tau+\tau^{\prime}\right| \sim \mu$. But, by the last line above this would imply that $\left|\tau+\tau^{\prime}\right| \lesssim O(\min \{c \mu, d\})$. Thus, as long as $c$ is chosen to be sufficiently small, we would have $\left|\tau+\tau^{\prime}\right| \ll \mu$, a contradiction. Therefore, throughout this paper, we will assume that $c$ has been chosen so small as to guarantee $\left|\tau+\tau^{\prime}\right| \lesssim\left|\xi+\xi^{\prime}\right|$ for convolutions of the type (93).

What the above calculations taken together show, is that one may replace the cutoff $s_{\mu, d}$ in the restriction (93) with a sum over the cutoffs $s_{\mu, d}^{\omega}$, and for each term in this sum one has that the two factors in the convolution are supported on antipodal blocks which differ by an angle at most $O\left(\sqrt{\frac{d}{\mu}}\right)$. Furthermore, the sum over $\omega$ is essentially diagonal with respect to these antipodal block pairs in that there is only one (essentially) pair of antipodal blocks for each $\omega$. The following diagram is useful for visualizing this:

We note here that by using the same computations as above, one can provide similar decompositions for expressions of the form $S_{\mu, \bullet \leqslant d}\left(S_{\lambda, \bullet \leqslant d} u \cdot \nabla S_{\lambda, d} v\right)$ and $S_{\mu, \bullet \leqslant d}\left(S_{\lambda, d} u \cdot \nabla S_{\lambda, \bullet<d} v\right)$, where $d$ is in the range $d<c \mu$ with $c \ll 1$ the fixed small number defined above. We recored all of these decompositions in the following: 

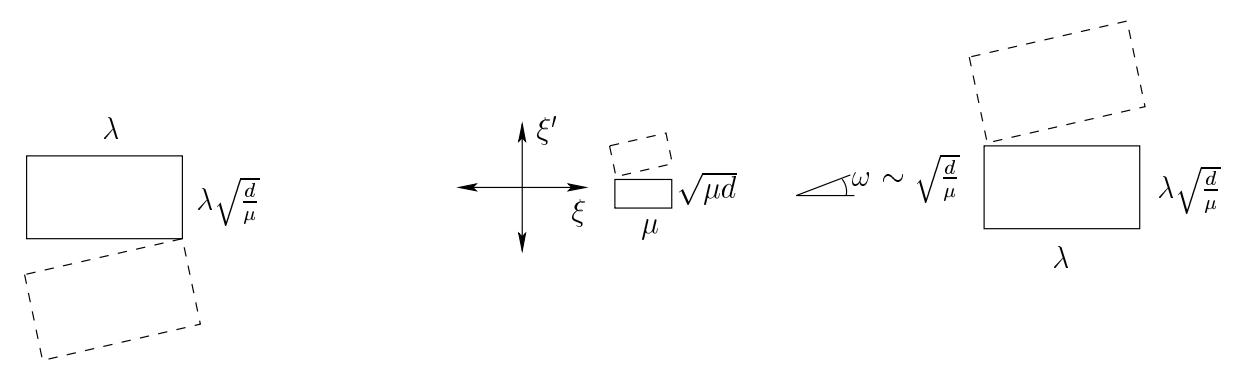

Figure 1. Spatial supports of multipliers in an angular decomposition.

Lemma 6.1 (High $\times$ High angular decomposition). For the expression:

$$
S_{\mu, d}\left(S_{\lambda, \bullet \leqslant \min \{d, c \mu\}} u \cdot \nabla S_{\lambda, \bullet \leqslant \min \{d, c \mu\}} v\right),
$$

one has the following angular decomposition:

$$
\begin{gathered}
s_{\mu, d}^{ \pm}\left(s_{\lambda, \bullet \leqslant \min \{d, c \mu\}}^{-} * s_{\lambda, \bullet \leqslant \min \{d, c \mu\}}^{+}\right), \\
=\sum_{\substack{\omega_{1}, \omega_{3}: \\
\left|\omega_{1} \mp \omega_{3}\right| \sim\left(\frac{d}{\mu}\right)^{\frac{1}{2}}}} s_{\mu, d}^{\omega_{1} \pm}\left(s_{\lambda, \bullet \leqslant \min \{d, c \mu\}}^{-} * b_{\left(\frac{d}{\mu}\right)^{\frac{1}{2}}}^{\omega_{3}} s_{\lambda, \bullet \leqslant \min \{d, c \mu\}}^{+}\right), \\
=\sum_{\substack{\omega_{1}, \omega_{2}, \omega_{3} \\
\left|\omega_{1} \mp \omega_{3}\right| \sim\left(\frac{d}{\mu}\right)^{\frac{1}{2}} \\
\left|\omega_{2}+\omega_{3}\right| \sim\left(\frac{d}{\mu}\right)^{\frac{1}{2}}}} s_{\mu, d}^{\omega_{1} \pm}\left(b_{\left(\frac{d}{\mu}\right)^{\frac{1}{2}}}^{\omega_{2}} s_{\lambda, \bullet \leqslant \min \{d, c \mu\}}^{-} * b_{\left(\frac{d}{\mu}\right)^{\frac{1}{2}}}^{\omega_{3}} s_{\lambda, \bullet \leqslant \min \{d, c \mu\}}^{+}\right),
\end{gathered}
$$

for the convolution of the associated cutoff functions in Fourier space. There is a similar decomposition for the terms $S_{\mu, \bullet \leqslant d}\left(S_{\lambda, \bullet \leqslant d} u \cdot \nabla S_{\lambda, d} v\right)$ and

$S_{\mu, \bullet \leqslant d}\left(S_{\lambda, d} u \cdot \nabla S_{\lambda, \bullet<d} v\right)$, where $d$ is in the range $d<c \mu$ and $c \ll 1$ is the small number fixed above.

Because the sum on the right hand side of the expression in the above lemma is essentially diagonal, we shall save notation in the sequel by abusively writing:

$$
\begin{gathered}
S_{\mu, d}\left(S_{\lambda, \bullet \leqslant \min \{d, c \mu\}} u \cdot \nabla S_{\lambda, \bullet \leqslant \min \{d, c \mu\}} v\right), \\
=\sum_{\omega} S_{\mu, d}^{ \pm \omega}\left(S_{\lambda, \bullet \leqslant \min \{d, c \mu\}} u \cdot \nabla B_{\left(\frac{d}{\mu}\right)^{\frac{1}{2}}}^{\omega} S_{\lambda, \bullet \leqslant \min \{d, c \mu\}} v\right), \\
=\sum_{\omega} S_{\mu, d}^{ \pm \omega}\left(B_{\left(\frac{d}{\mu}\right)^{\frac{1}{2}}}^{-\omega} S_{\lambda, \bullet \leqslant \min \{d, c \mu\}} u \cdot \nabla B_{\left(\frac{d}{\mu}\right)^{\frac{1}{2}}}^{\omega} S_{\lambda, \bullet \leqslant \min \{d, c \mu\}} v\right) .
\end{gathered}
$$


We shall also use this shorthand for the same decomposition applied to the terms $S_{\mu, \bullet \leqslant d}\left(S_{\lambda, \bullet \leqslant d} u \cdot \nabla S_{\lambda, d} v\right)$ and $S_{\mu, \bullet \leqslant d}\left(S_{\lambda, d} \cdot \nabla S_{\lambda, \bullet<d} v\right)$.

Next, we move on to several decompositions which are dual to Lemma (6.1). These take place in the presence of a Low $\times$ High frequency interaction. The first such decomposition will be used when the low frequency term controls the angles. The validity of this decomposition follows from essentially the same calculations as used for (94) and (95) above. For a proof, see [11. We record it here as:

Lemma 6.2 (Low $\times$ High wide angle decomposition). For the expression:

$$
S_{\lambda, \bullet<\min \{d, c \mu\}}\left(S_{\mu, d} u \nabla S_{\lambda, \bullet<\min \{d, c \mu\}} v\right),
$$

One has the following angular decomposition:

$$
\begin{aligned}
& s_{\lambda, \bullet<\min \{c \mu, d\}}^{+}\left(s_{\mu, d}^{ \pm} * s_{\lambda, \bullet<\min \{c \mu, d\}}^{+}\right) \\
&= \sum_{\substack{\omega_{1}, \omega_{2}, \omega_{3}: \\
\left|\omega_{1} \mp \omega_{2}\right| \sim\left(\frac{d}{\mu}\right)^{\frac{1}{2}} \\
\left|\omega_{1}-\omega_{3}\right| \sim\left(\frac{d}{\mu}\right)^{\frac{1}{2}}}} b_{\left(\frac{d}{\mu}\right)^{\frac{1}{2}}}^{\omega_{1}} s_{\lambda, \bullet<\min \{c \mu, d\}}^{+}\left(s_{\mu, d}^{\omega_{2} \pm} * b_{\left(\frac{d}{\mu}\right)^{\frac{1}{2}}}^{\omega_{3}} s_{\lambda, \bullet<\min \{c \mu, d\}}^{+}\right) . \\
& \quad
\end{aligned}
$$

for the convolution of the associated cutoff functions in Fourier space. There is a similar decomposition for the terms $S_{\lambda, \bullet \leqslant d}\left(S_{\mu, \bullet<d} u \nabla S_{\lambda, d} v\right)$ and

$S_{\lambda, d}\left(S_{\mu, \bullet \leqslant d} u \nabla S_{\lambda, \bullet<d} v\right)$ in the range $d<c \mu$, where $c \ll 1$ is a fixed small number.

We will also need a decomposition similar to that of Lemma 6.2 for the case where the high frequency term controls the angle. Again, for a proof see [1]. This is:

Lemma 6.3 (Low $\times$ High small angle decomposition). For the expression:

$$
S_{\lambda, d}^{\omega}\left(S_{\mu, \bullet \leqslant d} u \nabla S_{\lambda, \bullet<d} v\right),
$$

one has the following angular restriction:

$$
s_{\lambda, d}^{\omega_{1}+}\left(s_{\mu, \bullet \leqslant d}^{ \pm} * s_{\lambda, \bullet<d}^{+}\right)=s_{\lambda, d}^{\omega_{1}+}\left(s_{\mu, \bullet \leqslant d}^{\omega_{2}}{ }^{ \pm} * s_{\lambda, \bullet<d}^{\omega_{3}}{ }^{+}\right),
$$

for the convolution of the associated cutoff functions in Fourier space. Here the angles are restricted to the range $\left|\omega_{1}-\omega_{3}\right| \sim \sqrt{\frac{d}{\lambda}}$, and $\left|\omega_{1}- \pm \omega_{2}\right| \sim \sqrt{\frac{d}{\mu}}$.

Finally we record here the thickened version of Lemma 6.3 
Lemma 6.4 (Thickened Low $\times$ High small angle decomposition). For the expression:

$$
S_{\lambda, d}^{\omega}\left(S_{\mu} u \nabla S_{\lambda, \bullet<\mu \mu} v\right)
$$

one has the following angular restriction:

$$
s_{\lambda, d}^{\omega_{1}}+\left(s_{\mu}^{ \pm} * s_{\lambda, \bullet<c \mu}^{+}\right)=s_{\lambda, d}^{\omega_{1}+}\left(s_{\mu}^{ \pm} * b_{\left(\frac{d}{c \lambda}\right)^{\frac{1}{2}}}^{\omega_{3}} s_{\lambda, \bullet<c \mu}^{+}\right)
$$

for the convolution of the associated cutoff functions in Fourier space. Here the angles are restricted to the range $\left|\omega_{1}-\omega_{3}\right| \sim \sqrt{\frac{d}{c \lambda}}$.

As we have done above, in the sequel we shall write the decompositions in Lemmas 6.26 .4 using the following shorthand:

$S_{\lambda, \bullet<\min \{d, c \mu\}}\left(S_{\mu, d} u \cdot \nabla S_{\lambda, \bullet<\min \{d, c \mu\}} v\right)=$

$$
\begin{aligned}
& \sum_{\omega} B_{\left(\frac{d}{\mu}\right)^{\frac{1}{2}}}^{\omega} S_{\lambda, \bullet<\min \{d, c \mu\}}\left(S_{\mu, d}^{ \pm \omega} u \cdot \nabla B_{\left(\frac{d}{\mu}\right)^{\frac{1}{2}}}^{\omega} S_{\lambda, \bullet<\min \{d, c \mu\}} v\right), \\
& \sum_{\omega} S_{\lambda, d}^{\omega}\left(S_{\mu, \bullet \leqslant d} u \cdot \nabla S_{\lambda, d} v\right)= \sum_{\substack{\omega_{1}, \omega_{2}: \\
\left|\omega_{1} \mp \omega_{2}\right| \sim\left(\frac{d}{\mu}\right)^{\frac{1}{2}}}} S_{\lambda, d}^{\omega_{1}}\left(S_{\mu, \bullet \leqslant d}^{ \pm \omega_{2}} u \cdot \nabla S_{\lambda, d}^{\omega_{1}} v\right), \\
& \sum_{\omega} S_{\lambda, d}^{\omega}\left(S_{\mu} u \nabla S_{\lambda, \bullet<c \mu} v\right)= \sum_{\substack{\omega_{1}, \omega_{2}: \\
\left|\omega_{1}-\omega_{2}\right| \sim\left(\frac{d}{c \lambda}\right)^{\frac{1}{2}}}} S_{\lambda, d}^{\omega_{1}}\left(S_{\mu} u \nabla S_{\lambda, \bullet<c \mu}^{\omega_{2}} v\right) .
\end{aligned}
$$

It is important to note that the sum on the right hand side of (97) above is not diagonal in the two angles $\omega_{1}$ and $\omega_{2}$. This is one of the main reasons why we need to employ the extra flexibility in the norms (666). Also, while the sum on the right hand side of (98) is essentially diagonal for a fixed small $c \ll 1$, we have elected to keep the more precise form because we will need to pick a $c$ based on the other implicit constants which appear in various decompositions in the proof.

\section{Frequency Decomposition of the Nonlinearity}

The remainder of the paper is devoted to the proof of Theorem 1.1. This will be done through Picard iterating the integral equation:

$$
\phi=W(f, g)+\square^{-1}(\phi \nabla \phi),
$$


in the spaces $G_{\Omega}^{1}$ and $F_{\Omega}^{s, 2} \cap G_{\Omega}^{1}$ for $1<s$. Due to the quadratic nature of the nonlinearity, it suffices to prove the following:

Theorem 7.1 (Solution of the division problem). For $n=4$, the $F_{\Omega}$ and $G_{\Omega}$ spaces solve the division problem for the system (1) in the sense that one has the following bilinear estimates for functions $u$ and $v$ :

$$
\begin{aligned}
\left\|\square^{-1}(u \nabla v)\right\|_{G_{\Omega}^{1}} & \lesssim\|u\|_{G_{\Omega}^{1}}\|v\|_{G_{\Omega}^{1}}, \\
\left\|\square^{-1}(u \nabla v)\right\|_{F_{\Omega}^{s, 2}} & \lesssim\|u\|_{G_{\Omega}^{1}}\|v\|_{F_{\Omega}^{s, 2}}+\|u\|_{F_{\Omega}^{s, 2}}\|v\|_{G_{\Omega}^{1}} .
\end{aligned}
$$

In what follows, we shall concentrate solely on the estimate (100). The other, estimate (101), will follow directly from the dyadic estimates employed in the proof of (100).

Our first step is the usual Littlewood-Paley decomposition of the nonlinear term $\square^{-1}(u \nabla v)$ with respect to space-time frequencies:

$$
\square^{-1}(u \nabla v)=\sum_{\lambda_{i}} \square^{-1}\left(S_{\lambda_{1}} u \nabla S_{\lambda_{2}} v\right) .
$$

We now split the sum on the right hand side of (102) into the three cases: $\lambda_{1} \sim \lambda_{2}$, $\lambda_{1} \ll \lambda_{2}$, and $\lambda_{2} \ll \lambda_{1}$. In the sequel, we only concentrate on the first two interactions, the last case being similar to the second through some standard "weight trading". Taking into account the $\ell^{1}$ Besov structure of the $G_{\Omega}^{1}$ space, to prove (100), it suffices to show the following two bilinear estimates:

$\sum_{\mu: \mu \lesssim \max \left\{\lambda_{1}, \lambda_{2}\right\}} \mu\left\|\square^{-1}\left(S_{\lambda_{1}} u \nabla S_{\lambda_{2}} v\right)\right\|_{G_{\Omega, \mu}} \lesssim \lambda_{1} \lambda_{2}\|u\|_{F_{\Omega, \lambda_{1}}}\|v\|_{F_{\Omega, \lambda_{2}}}, \lambda_{1} \sim \lambda_{2}$,

$$
\left\|\square^{-1}\left(S_{\mu} u \nabla S_{\lambda} v\right)\right\|_{G_{\Omega, \lambda}} \lesssim \mu\|u\|_{G_{\Omega, \mu}}\|v\|_{F_{\Omega, \lambda}}, \mu \ll \lambda
$$

Due to the fact that both of the above estimates are scale invariant, it suffices to prove them for $\lambda_{1}=\lambda_{2}=1$, and $\mu=1$ respectively. In the next two subsections, we shall break these estimates down further into a series of cases involving the various function spaces that make up the $G_{\Omega, \lambda}$ and $F_{\Omega, \lambda}$ spaces. These estimates will be placed in highlighted format for the convenience of the reader. Each individual estimate will then be proved separately in the remaining two sections of the paper.

7.1. High $\times$ High regime: List of dyadic estimates corresponding to (103). In what follows, we will not explicitly take angular derivatives of any of the expressions we are to estimate. Instead, we will prove bilinear estimates where at most 
one term in the product ${ }^{6}$ on the right hand side contains a norm involving angular derivatives. Thus, using the Leibniz rule, one can safely add an angular derivative to all estimates that follow below to get estimate (103) above.

We begin by further decomposing the expression $S_{\mu} \square^{-1}\left(S_{1} u \nabla S_{1} v\right), \mu \lesssim 1$. Using the (approximate) idempotence of $S_{\mu}$, we first compute that:

$$
S_{\mu} \square^{-1}\left(S_{1} u \nabla S_{1} v\right)=\square^{-1} S_{\mu}\left(S_{1} u \nabla S_{1} v\right)+S_{\mu}\left[S_{\mu}, \square^{-1}\right]\left(S_{1} u \nabla S_{1} v\right) .
$$

To compute the commutator term, let $H_{\bullet} \lesssim 1$ be a function with space-time frequency $\lesssim 1$. Then we have that:

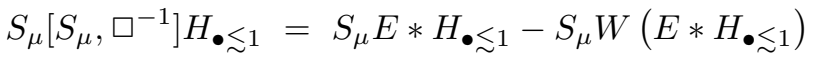

$$
\begin{aligned}
& -S_{\mu} E * S_{\mu} H_{\bullet} \leqslant 1+S_{\mu} W\left(E * S_{\mu} H_{\bullet} \leq 1\right), \\
& =-\sum_{\substack{\sigma \\
\mu \leqslant \sigma \lesssim 1}} S_{\mu} W\left(E * S_{\sigma} H_{\bullet} \lesssim 1\right)+S_{\mu} W\left(E * S_{\mu} H_{\bullet} \lesssim 1\right) \text {, } \\
& =-\sum_{\substack{\sigma: \\
\mu<\sigma \lesssim 1}} S_{\mu} W\left(E * S_{\sigma} H_{\bullet} \leqslant 1\right) \\
& =-\sum_{\substack{\sigma: \vdots \\
\mu<\sigma \lesssim 1}} W\left(P_{\mu} E * S_{\sigma, \sigma} H_{\bullet} \leq 1\right) .
\end{aligned}
$$

Therefore, we have that:

$$
S_{\mu} \square^{-1}\left(S_{1} u \nabla S_{1} v\right)=\square^{-1} S_{\mu}\left(S_{1} u \nabla S_{1} v\right)-\sum_{\sigma: \mu<\sigma \lesssim 1} W\left(P_{\mu} S_{\sigma, \sigma} \frac{1}{\Xi}\left(S_{1} u \nabla S_{1} v\right)\right) .
$$

To handle the second term on line (105) it is enough to prove the estimate:

$$
\sum_{\mu \lesssim 1} \mu\left\|\sum_{\sigma: \mu<\sigma \lesssim 1}\left(P_{\mu} S_{\sigma, \sigma} \frac{1}{\Xi}\left(S_{1} u \nabla S_{1} v\right)\right)\right\|_{L^{\infty}\left(L^{2}\right)} \lesssim\|u\|_{F_{1}}\|v\|_{F_{1}}
$$

Next, we move on to the first term on the right hand side of (105) above. For this we fix a small $c \ll 1$ as explained in Section [ 6 and write:

$$
\begin{gathered}
S_{\mu}\left(S_{1} u \nabla S_{1} v\right)=S_{\mu}\left(S_{1} u \nabla S_{1, c \mu \leqslant \bullet} v\right)+S_{\mu}\left(S_{1, c \mu \leqslant \bullet} u \nabla S_{1, \bullet<c \mu} v\right) \\
+S_{\mu}\left(S_{1, \bullet<c \mu} u \nabla S_{1, \bullet<c \mu} v\right) .
\end{gathered}
$$

In all that follows here, we will only estimate the terms of (107) in the $X$ and $Y$ spaces defined on lines (47) and (48) respectively. The addition of the $L^{\infty}\left(L^{2}\right)$

\footnotetext{
${ }^{6}$ With the exception of estimate 115 below which involves angular derivatives of both terms in the product. Notice that this is acceptable because the norm 68 does not involve any angular derivatives.
} 
estimate, included on line (49), follows from estimates on these first two norms in a standard way. See [1] for details. To control the fist two terms on the right hand side of (107), we will prove that:

$$
\begin{aligned}
\sum_{\mu}\left\|S_{\mu}\left(S_{1} u \nabla S_{1, c \mu \leqslant \bullet} v\right)\right\|_{L^{1}\left(L^{2}\right)} & \lesssim\|u\|_{F_{1}}\|v\|_{F_{1}}, \\
\sum_{\mu}\left\|S_{\mu}\left(S_{1, c \mu \leqslant \bullet} u \nabla S_{1, \bullet<c \mu} v\right)\right\|_{L^{1}\left(L^{2}\right)} & \lesssim\|u\|_{F_{1}}\|v\|_{F_{1}} .
\end{aligned}
$$

It remains to estimate the terms $\square^{-1} S_{\mu}\left(S_{1, \bullet<c \mu} u \nabla S_{1, \bullet<c \mu} v\right)$. To do this we decompose these expressions with respect to all possible dyadic distances from the light cone in Fourier space. We group this sum together as follows:

$$
\begin{aligned}
S_{\mu}\left(S_{1, \bullet<c \mu} u \nabla S_{1, \bullet<c \mu} v\right)= & \sum_{\substack{d, \delta_{1}, \delta_{2}: \\
\delta_{1}<c \mu \\
\delta_{2}<c \mu}} S_{\mu, d}\left(S_{1, \delta_{1}} u \nabla S_{1, \delta_{2}} v\right), \\
= & \sum_{\substack{d: d<c \mu \\
\text { (110) }}} S_{\mu, \bullet \leqslant d}\left(S_{1, \bullet \leqslant d} u \nabla S_{1, d} v\right) \\
& \quad+\sum_{d: d<c \mu} S_{\mu, \bullet \leqslant d}\left(S_{1, d} u \nabla S_{1, \bullet<d} v\right) \\
& \quad+\sum_{d} S_{\mu, d}\left(S_{1, \bullet<\min \{d, c \mu\}} u \nabla S_{1, \bullet<\min \{d, c \mu\}} v\right) .
\end{aligned}
$$

For the first term on the right hand side of (110), we will prove the two estimates:

$$
\begin{aligned}
& \sum_{\mu}\left\|\sum_{d: d<c \mu} S_{\mu, \bullet \leqslant d}\left(S_{1, \bullet \leqslant d} u \nabla S_{1, d} v\right)\right\|_{L^{1}\left(L^{2}\right)} \lesssim\|u\|_{F_{\Omega, 1}}\|v\|_{F_{1}}, \\
& \sum_{\mu}\left\|\sum_{d: d<c \mu} S_{\mu, \bullet \leqslant d}\left(S_{1, \bullet \leqslant d} u \nabla S_{1, d} v\right)\right\|_{L^{1}\left(L^{2}\right)} \lesssim\|u\|_{F_{1}}\|v\|_{F_{\Omega, 1}} .
\end{aligned}
$$

Notice that the proof of estimate (103) for the second term in line (110) follows from the proof of (111)-(112) and some weight trading. It remains to deal with the last term of line (110). In this case we'll need to rely on the $L^{2}$ based norms. Using some weight trading, this can be reduced to the single estimate: 
(113)

$$
\sum_{\mu, d} d^{-\frac{1}{2}}\left\|S_{\mu, d}\left(S_{1, \bullet<\min \{d, c \mu\}} u \nabla S_{1, \bullet<\min \{d, c \mu\}} v\right)\right\|_{L^{2}\left(L^{2}\right)} \lesssim\|u\|_{F_{\Omega, 1}}\|v\|_{F_{1}} .
$$

Because of the limitations of the $L^{2}$ based spaces (i.e. that they do not have an analog of (89)-(90)), we also need to recover the $Z$ norms by hand for this frequency interaction. It suffices to be able to bound the norms (67) and (68). Using the multiplier boundedness lemma (4.2) and some weight trading where necessary, this reduces to showing the two estimates:

$$
\begin{aligned}
& \sum_{\mu, d} \frac{1}{\mu^{\frac{3}{2}} d^{\frac{1}{2}}} \int\left(\sum_{\omega}\left\|S_{\mu, d}^{\omega}\left(S_{1, \bullet<\min \{d, c \mu\}} u \nabla S_{1, \bullet<\min \{d, c \mu\}} v\right)(t)\right\|_{L_{x}^{\infty}}^{2}\right)^{\frac{1}{2}} d t \\
& \lesssim\|u\|_{F_{\Omega, 1}}\|v\|_{F_{1}},
\end{aligned}
$$

$$
\begin{aligned}
\sum_{\mu, d} \frac{1}{\mu d} \int \sup _{\omega}\left\|S_{\mu, d}^{\omega}\left(S_{1, \bullet<\min \{d, c \mu\}} u \nabla S_{1, \bullet<\min \{d, c \mu\}} v\right)(t)\right\|_{L_{x}^{\infty}} d t & \\
& \lesssim\|u\|_{F_{\Omega, 1}}\|v\|_{F_{\Omega, 1}} .
\end{aligned}
$$

7.2. Low $\times$ High regime: List of dyadic estimates corresponding to (104). Here we follow the same procedure as in the previous section, proving bilinear estimates where one term in the product can safely absorb an angular derivative. Of course there is an exception for estimates (120) and (128) below, which do not need to absorb any angular derivatives.

Our first order of business here is to note that, due to the fact $1 \ll \lambda$, one has the identity:

$$
S_{\lambda} \square^{-1}\left(S_{1} u \nabla S_{\lambda} v\right)=S_{\lambda} \square^{-1} S_{\lambda}\left(S_{1} u \nabla S_{\lambda} v\right) .
$$

Because of this, we can avoid proving an extra estimate of the form (106) here. We now use a rough decomposition to isolate things so they are sufficiently close to the light cone in Fourier space. For this purpose we fix a small $c \ll 1$ as described in 
Section [6] and write:

$$
\begin{gathered}
S_{\lambda}\left(S_{1} u \nabla S_{\lambda} v\right)=S_{\lambda}\left(S_{1} u \nabla S_{\lambda, c \leqslant \bullet} v\right)+S_{\lambda, c \leqslant \bullet}\left(S_{1} u \nabla S_{\lambda, \bullet<c} v\right) \\
+S_{\lambda, \bullet<c}\left(S_{1} u \nabla S_{\lambda, \bullet<c} v\right) .
\end{gathered}
$$

Notice that this decomposition is dual to that of (107). Again, as we mentioned in the previous subsection, we will only concern ourselves with estimating the $X$ and $Y$ space portion of the norm (49), as the $L^{\infty}\left(L^{2}\right)$ type estimate follows once we have done this. In order to estimate $\square^{-1}$ of the first two terms on the right hand side of (116) in the $F_{\Omega, \lambda}$ spaces, it is enough to prove the following two estimates:

$$
\begin{aligned}
\left\|S_{\lambda}\left(S_{1} u \nabla S_{\lambda, c \leqslant \bullet} v\right)\right\|_{L^{1}\left(L^{2}\right)} & \lesssim\|u\|_{F_{1}} \cdot \lambda\|v\|_{F_{\lambda}}, \\
\sum_{c \leqslant d} d^{-\frac{1}{2}}\left\|S_{\lambda, d}\left(S_{1} u \nabla S_{\lambda, \bullet<c} v\right)\right\|_{L^{2}\left(L^{2}\right)} & \lesssim\|u\|_{F_{1}} \cdot \lambda\|v\|_{F_{\lambda}} .
\end{aligned}
$$

We also need to recover the norms (67) and (68) by hand for the second term on the right hand side of (116). To do this we will show that:

$$
\begin{aligned}
& \sum_{c \leqslant d} \frac{1}{\lambda^{\frac{3}{2}} d^{\frac{1}{2}}} \int\left(\sum_{\omega}\left\|S_{\lambda, d}^{\omega}\left(S_{1} u \nabla S_{\lambda, \bullet<c} v\right)(t)\right\|_{L_{x}^{\infty}}^{2}\right)^{\frac{1}{2}} d t \lesssim\|u\|_{F_{1}} \cdot \lambda\|v\|_{F_{\lambda}}, \\
& \sum_{c \leqslant d} \frac{1}{\lambda d} \int \sup _{\omega}\left\|S_{\lambda, d}^{\omega}\left(S_{1} u \nabla S_{\lambda, \bullet<c} v\right)(t)\right\|_{L_{x}^{\infty}} d t \lesssim\|u\|_{F_{1}} \cdot \lambda\|v\|_{F_{\Omega, \lambda}} .
\end{aligned}
$$


At this point, we are in a position to deal with the last term on the right hand side of (116). We further decompose this in a manner dual to (110):

$$
\begin{aligned}
S_{\lambda, \bullet<c}\left(S_{1} u \nabla S_{\lambda, \bullet<c} v\right)= & \sum_{\substack{d, \delta_{1}, \delta_{2} \\
d<c \\
\delta_{2}<c}} S_{\lambda, d}\left(S_{1, \delta_{1}} u \nabla S_{\lambda, \delta_{2}} v\right), \\
= & \sum_{d<c} S_{\lambda, \bullet \leqslant d}\left(S_{1, \bullet \leqslant d} u \nabla S_{\lambda, d} v\right) \\
& \quad+\sum_{d<c} S_{\lambda, d}\left(S_{1, \bullet \leqslant d} u \nabla S_{\lambda, \bullet<d} v\right) \\
& \quad+\sum_{d} S_{\lambda, \bullet<\min \{d, c\}}\left(S_{1, d} u \nabla S_{\lambda, \bullet \min \{d, c\}} v\right) .
\end{aligned}
$$

To deal with the first term on the right hand side of (121), we'll prove the two separate estimates:

$$
\begin{aligned}
\left\|\sum_{d<c} S_{\lambda, \bullet \leqslant d}\left(S_{1, \bullet \leqslant d} u \nabla S_{\lambda, d} v\right)\right\|_{L^{1}\left(L^{2}\right)} & \lesssim\|u\|_{F_{1}} \cdot \lambda\|v\|_{F_{\Omega, \lambda}}, \\
\left\|\sum_{d<c} S_{\lambda, \bullet \leqslant d}\left(S_{1, \bullet \leqslant d} u \nabla S_{\lambda, d} v\right)\right\|_{L^{1}\left(L^{2}\right)} & \lesssim\|u\|_{F_{\Omega, 1}} \cdot \lambda\|v\|_{F_{\lambda}},
\end{aligned}
$$

To show the second term on the right hand side of (121) is in the $F_{\Omega, \lambda}$ spaces, we prove the pair of estimates:

$$
\begin{aligned}
& \sum_{d<c} d^{-\frac{1}{2}}\left\|S_{\lambda, d}\left(S_{1, \bullet \leqslant d} u \nabla S_{\lambda, \bullet<d} v\right)\right\|_{L^{2}\left(L^{2}\right)} \lesssim\|u\|_{F_{1}} \cdot \lambda\|v\|_{F_{\Omega, \lambda}}, \\
& \sum_{d<c} d^{-\frac{1}{2}}\left\|S_{\lambda, d}\left(S_{1, \bullet \leqslant d} u \nabla S_{\lambda, \bullet<d} v\right)\right\|_{L^{2}\left(L^{2}\right)} \lesssim\|u\|_{F_{\Omega, 1}} \cdot \lambda\|v\|_{F_{\lambda}},
\end{aligned}
$$

Next, we will need to recover the special $L^{1}\left(L^{\infty}\right)$ norms for the second term on the right hand side of (121). Our first estimate will used for controlling the expression (121) when the low frequency term in the product need to absorb an angular derivative. This is where we will need to use the more complicated version, (66), of the $Z$ norms. Here, the $u^{\alpha}$ in the sum (62) will come from the terms in the 
following sum:

$$
S_{\lambda}\left(S_{\bullet \ll \lambda} u \nabla S_{\lambda} v\right)=\sum_{\mu: \mu \ll \lambda} S_{\mu} u \nabla S_{\lambda} v .
$$

Due to the Besov structure in the $G$ space, we only need to deal with a single term of this. By rescaling, as we have done throughout this section, we may assume that $\mu=1$. For this fixed piece, we shall use the angle set where $\left|\theta_{d}\right|=d^{\frac{1}{2}}$. Therefore, it will suffice to prove:

$$
\begin{aligned}
\sum_{d} \frac{1}{\lambda d^{\frac{1}{2}}} \int\left(\sum_{\substack{\theta: \\
|\theta| \sim d^{\frac{1}{2}}}} \sup _{\omega \subseteq \theta}\left\|S_{\lambda, \bullet \min \{d, c\}}^{\omega}\left(S_{1, d} u \nabla S_{\lambda, \bullet \min \{d, c\}} v\right)(t)\right\|_{L_{x}^{\infty}}^{2}\right)^{\frac{1}{2}} d t \\
\\
\lesssim\|u\|_{F_{1}} \cdot \lambda\|v\|_{F_{\Omega, \lambda}}
\end{aligned}
$$

When estimating the second term of the expression (121) where an angular derivative falls on the high frequency term in the product, we shall use the simpler norm (67). Finally, we need to recover the norm (68). Therefore, we shall prove the following two estimates:

$$
\begin{array}{r}
\sum_{d} \frac{1}{\lambda^{\frac{3}{2}} d^{\frac{1}{2}}} \int\left(\sum_{\omega}\left\|S_{\lambda, \bullet \min \{d, c\}}^{\omega}\left(S_{1, d} u \nabla S_{\lambda, \bullet \min \{d, c\}} v\right)(t)\right\|_{L_{x}^{\infty}}^{2}\right)^{\frac{1}{2}} d t \\
\lesssim\|u\|_{F_{\Omega, 1}} \cdot \lambda\|v\|_{F_{\lambda}},
\end{array}
$$

$$
\begin{aligned}
\sum_{d} \frac{1}{\lambda d} \int \sup _{\omega}\left\|S_{\lambda, \bullet<\min \{d, c\}}^{\omega}\left(S_{1, d} u \nabla S_{\lambda, \bullet<\min \{d, c\}} v\right)(t)\right\|_{L_{x}^{\infty}} d t & \\
& \lesssim\|u\|_{F_{\Omega, 1}} \cdot \lambda\|v\|_{F_{\Omega, \lambda}},
\end{aligned}
$$

It remains to deal with the last term on the right hand side of (121). For this we'll prove the following two estimates involving the $Z_{1}$ and $Z_{\Omega, 1}$ spaces: 


$$
\left\|\sum_{d} S_{\lambda, \bullet<\min \{d, c\}}\left(S_{1, d} u \nabla S_{\lambda, \bullet<\min \{d, c\}} v\right)\right\|_{L^{1}\left(L^{2}\right)} \lesssim\|u\|_{Z_{1}} \cdot \lambda\|v\|_{F_{\Omega, \lambda}},
$$

$$
\left\|\sum_{d} S_{\lambda, \bullet \min \{d, c\}}\left(S_{1, d} u \nabla S_{\lambda, \bullet<\min \{d, c\}} v\right)\right\|_{L^{1}\left(L^{2}\right)} \lesssim\|u\|_{Z_{\Omega, 1}} \cdot \lambda\|v\|_{F_{\lambda}} .
$$

\section{Inductive Estimates I. High $\times$ High Frequency Interactions}

We now begin the proof of the boxed estimates in section 7.1 To streamline the process, we will list out the individual steps in the proofs all in a row with very little text explanations in between. At the beginning of each block of estimates, we list in order of use the various Sobolev, Strichartz, and multiplier boundedness estimates that are used in the lines that follow. It would have been more convenient to include these references on the lines where they are used, but there does not seem to be enough room in the typesetting to allow this.

proof of estimate (106). For a fixed $\sigma$ and $\mu$ we use the multiplier boundedness Lemma 4.1 the localized Sobolev estimate 84), Hölders inequality, and the energy estimate (51) to compute that:

$$
\begin{aligned}
&\left\|S_{\sigma, \sigma} \frac{1}{\Xi} P_{\mu}\left(S_{1} u \nabla S_{1} v\right)\right\|_{L^{\infty}\left(L^{2}\right)}, \\
& \lesssim \sigma^{-2}\left\|P_{\mu}\left(S_{1} u \nabla S_{1} v\right)\right\|_{L^{\infty}\left(L^{2}\right)}, \\
& \lesssim\left(\frac{\mu}{\sigma}\right)^{2}\left\|S_{1} u \nabla S_{1} v\right\|_{L^{\infty}\left(L^{1}\right)} \\
& \lesssim\left(\frac{\mu}{\sigma}\right)^{2}\left\|S_{1} u\right\|_{L^{\infty}\left(L^{2}\right)} \cdot\left\|\nabla S_{1} v\right\|_{L^{\infty}\left(L^{2}\right)} \\
& \lesssim\left(\frac{\mu}{\sigma}\right)^{2}\|u\|_{F_{1}} \cdot\|v\|_{F_{1}} .
\end{aligned}
$$

Multiplying this last expression by $\mu$ and then summing yields:

$$
\begin{aligned}
\text { (L.H.S.) 106) } & \lesssim \sum_{\mu \lesssim 1} \mu \sum_{\mu<\sigma}\left(\frac{\mu}{\sigma}\right)^{2}\|u\|_{F_{1}} \cdot\|v\|_{F_{1}} \\
& \lesssim\|u\|_{F_{1}} \cdot\|v\|_{F_{1}} .
\end{aligned}
$$

proof of estimates (108)- (109). Due to the boundedness of the multiplier $S_{1, \bullet<c \mu}$ on the $F$ spaces, and the ability to trade the $\nabla$ weight between either term of the product, it suffices to prove the first estimate (108). We begin by noting that:

$$
\text { (L.H.S.) (108) } \lesssim \sum_{\substack{\sigma, \mu \\ \sigma \leqslant \mu \lesssim 1}} \| P_{\sigma} S_{\mu}\left(S_{1} u \nabla S_{1, c \mu \leqslant \bullet} \cdot \|_{L^{1}\left(L^{2}\right)} .\right.
$$


We now fix both $\sigma$ and $\mu$ for the moment and use the local Sobolev estimate (82), Hölders inequality, the Strichartz estimate (53), and the $L^{2}$ estimate (61) to compute that:

$$
\begin{array}{cc} 
& \left\|P_{\sigma} S_{\mu}\left(S_{1} u \nabla S_{1, c \mu \leqslant \bullet} v\right)\right\|_{L^{1}\left(L^{2}\right)}, \\
\lesssim & \sigma^{\frac{2}{3}}\left\|S_{1} u \nabla S_{1, c \mu \leqslant \bullet} v\right\|_{L^{1}\left(L^{\frac{3}{2}}\right)}, \\
\lesssim & c^{-\frac{1}{2}} \sigma^{\frac{1}{6}}\left(\frac{\sigma}{\mu}\right)^{\frac{1}{2}}\left\|S_{1} u\right\|_{L^{2}\left(L^{6}\right)} \cdot(c \mu)^{\frac{1}{2}}\left\|\nabla S_{1, c \mu \leqslant \bullet} v\right\|_{L^{2}\left(L^{2}\right)}, \\
\lesssim & c^{-\frac{1}{2}} \sigma^{\frac{1}{6}}\left(\frac{\sigma}{\mu}\right)^{\frac{1}{2}}\|u\|_{F_{1}} \cdot\|v\|_{F_{1}} .
\end{array}
$$

This last expression can now be summed over $\sigma$ and $\mu$ to yield:

$$
\begin{aligned}
\text { (L.H.S.) (108) } & \lesssim \sum_{\sigma \lesssim 1} \sum_{\sigma \leqslant \mu} c^{-\frac{1}{2}} \sigma^{\frac{1}{6}}\left(\frac{\sigma}{\mu}\right)^{\frac{1}{2}}\|u\|_{F_{1}} \cdot\|v\|_{F_{1}} \\
& \lesssim c^{-\frac{1}{2}}\|u\|_{F_{1}} \cdot\|v\|_{F_{1}} .
\end{aligned}
$$

For a fixed small $c$, this yields the desired result.

proof of estimate (111). We begin by fixing both $\mu$ and $d$. Next, we use in order the angular decomposition (95), the multiplier Lemma 4.1 and orthogonality, the local Sobolev estimate (83), the angular concentration estimate (86), and finally the Strichartz estimate (54) as well as the $L^{2}$ estimate (60) to compute that:

$$
\begin{aligned}
& \int\left\|S_{\mu, \bullet \leqslant d}\left(S_{1, \bullet \leqslant d} u \nabla S_{1, d} v\right)(t)\right\|_{L_{x}^{2}} d t, \\
& =\quad \int\left\|\sum_{\omega} S_{\mu, \bullet \leqslant d}^{ \pm \omega}\left(B_{\left(\frac{d}{\mu}\right)^{\frac{1}{2}}}^{-\omega} S_{1, \bullet \leqslant d} u \nabla B_{\left(\frac{d}{\mu}\right)^{\frac{1}{2}}}^{\omega} S_{1, d} v\right)(t)\right\|_{L_{x}^{2}} d t, \\
& \lesssim \quad \int\left(\sum_{\omega}\left\|B_{\left(\frac{d}{\mu}\right)^{\frac{1}{2}}}^{ \pm \omega} P_{\mu}\left(B_{\left(\frac{d}{\mu}\right)^{\frac{1}{2}}}^{-\omega} S_{1, \bullet \leqslant d} u \nabla B_{\left(\frac{d}{\mu}\right)^{\frac{1}{2}}}^{\omega} S_{1, d} v\right)(t)\right\|_{L_{x}^{2}}^{2}\right)^{\frac{1}{2}} d t \\
& \lesssim \quad \mu^{\frac{5}{6}-} d^{\frac{1}{2}-} \int\left(\sum_{\omega}\left\|\left(B_{\left(\frac{d}{\mu}\right)^{\frac{1}{2}}}^{-\omega} S_{1, \bullet \leqslant d} u \nabla B_{\left(\frac{d}{\mu}\right)^{\frac{1}{2}}}^{\omega} S_{1, d} v\right)(t)\right\|_{L_{x}^{\frac{6}{5}+}}^{2}\right)^{\frac{1}{2}} d t, \\
& \lesssim \quad \mu^{\frac{5}{6}-} d^{\frac{1}{2}-} \int \sup _{\omega}\left\|B_{\left(\frac{d}{\mu}\right)^{\frac{1}{2}}}^{-\omega} S_{1, \bullet \leqslant d} u(t)\right\|_{L_{x}^{3+}} \cdot\left(\sum_{\omega}\left\|\nabla B_{\left(\frac{d}{\mu}\right)^{\frac{1}{2}}}^{\omega} S_{1, d} v(t)\right\|_{L_{x}^{2}}^{2}\right)^{\frac{1}{2}} d t, \\
& \lesssim \quad \mu^{\frac{7}{12}-} d^{\frac{3}{4}-} \int\left\|S_{1, \bullet \leqslant d}\langle\Omega\rangle^{\frac{1}{2}} u(t)\right\|_{L_{x}^{3+}} \cdot\left\|\nabla S_{1, d} v(t)\right\|_{L_{x}^{2}} d t, \\
& \lesssim \mu^{\frac{5}{6}-}\left(\frac{d}{\mu}\right)^{\frac{1}{4}-}\left\|S_{1, \bullet \leqslant d}\langle\Omega\rangle^{\frac{1}{2}} u\right\|_{L^{2}\left(L^{3+}\right)} \cdot d^{\frac{1}{2}}\left\|\nabla S_{1, d} v\right\|_{L^{2}\left(L^{2}\right)} \\
& \lesssim \mu^{\frac{5}{6}-}\left(\frac{d}{\mu}\right)^{\frac{1}{4}-}\|u\|_{F_{\Omega, 1}} \cdot\|v\|_{F_{1}} \text {. }
\end{aligned}
$$


This last expression can now be safely summed over both $\mu$ and $d$ to yield:

$$
\begin{aligned}
\text { (L.H.S.) 111) } & \lesssim \sum_{\substack{\mu, d: \\
\mu \lesssim 1, d<c \mu}} \mu^{\frac{5}{6}-}\left(\frac{d}{\mu}\right)^{\frac{1}{4}-}\|u\|_{F_{1}} \cdot\|v\|_{F_{\Omega, 1}}, \\
& \lesssim\|u\|_{F_{\Omega, 1}} \cdot\|v\|_{F_{1}} .
\end{aligned}
$$

proof of estimate (112). We begin by fixing both $\mu$ and $d$, we use in order the angular decomposition (95), the multiplier Lemma 4.1 and orthogonality, the local Sobolev estimate (82), the angular concentration estimate [87), and finally the Strichartz estimate (53) as well as the $L^{2}$ estimate (60) to compute that:

$$
\begin{aligned}
& \int\left\|S_{\mu, \bullet \leqslant d}\left(S_{1, \bullet \leqslant d} u \nabla S_{1, d} v\right)(t)\right\|_{L_{x}^{2}} d t \\
& =\quad \int\left\|\sum_{\omega} S_{\mu, \bullet \leqslant d}^{ \pm \omega}\left(B_{\left(\frac{d}{\mu}\right)^{\frac{1}{2}}}^{-\omega} S_{1, \bullet \leqslant d} u \nabla B_{\left(\frac{d}{\mu}\right)^{\frac{1}{2}}}^{\omega} S_{1, d} v\right)(t)\right\|_{L_{x}^{2}} d t, \\
& \lesssim \quad \int\left(\sum_{\omega}\left\|B_{\left(\frac{d}{\mu}\right)^{\frac{1}{2}}}^{ \pm \omega} P_{\mu}\left(B_{\left(\frac{d}{\mu}\right)^{\frac{1}{2}}}^{-\omega} S_{1, \bullet \leqslant d} u \nabla B_{\left(\frac{d}{\mu}\right)^{\frac{1}{2}}}^{\omega} S_{1, d} v\right)(t)\right\|_{L_{x}^{2}}^{2}\right)^{\frac{1}{2}} d t, \\
& \lesssim \quad \mu^{\frac{5}{12}} d^{\frac{1}{4}} \int\left(\sum_{\omega}\left\|\left(B_{\left(\frac{d}{\mu}\right)^{\frac{1}{2}}}^{-\omega} S_{1, \bullet \leqslant d} u \nabla B_{\left(\frac{d}{\mu}\right)^{\frac{1}{2}}}^{\omega} S_{1, d} v\right)(t)\right\|_{L_{x}^{\frac{3}{2}}}^{2}\right)^{\frac{1}{2}} d t \\
& \lesssim \quad \mu^{\frac{5}{12}} d^{\frac{1}{4}} \int\left(\sum_{\omega}\left\|B_{\left(\frac{d}{\mu}\right)^{\frac{1}{2}}}^{-\omega} S_{1, \bullet \leqslant d} u(t)\right\|_{L_{x}^{6}}^{2}\right)^{\frac{1}{2}} \cdot \sup _{\omega}\left\|\nabla B_{\left(\frac{d}{\mu}\right)^{\frac{1}{2}}}^{\omega} S_{1, d} v(t)\right\|_{L_{x}^{2}} d t, \\
& \lesssim \quad \mu^{-\frac{1}{12}} d^{\frac{3}{4}} \int\left(\sum_{\omega}\left\|B_{\left(\frac{d}{\mu}\right)^{\frac{1}{2}}}^{-\omega} S_{1, \bullet \leqslant d} u(t)\right\|_{L_{x}^{6}}^{2}\right)^{\frac{1}{2}} \cdot\left\|\nabla S_{1, d}\langle\Omega\rangle v(t)\right\|_{L_{x}^{2}} d t, \\
& \lesssim \mu^{\frac{1}{6}}\left(\frac{d}{\mu}\right)^{\frac{1}{4}}\left(\sum_{\omega}\left\|B_{\left(\frac{d}{\mu}\right)^{\frac{1}{2}}}^{-\omega} S_{1, \bullet \leqslant d} u\right\|_{L^{2}\left(L^{6}\right)}^{2}\right)^{\frac{1}{2}} \cdot d^{\frac{1}{2}}\left\|\nabla S_{1, d}\langle\Omega\rangle v\right\|_{L^{2}\left(L^{2}\right)}, \\
& \lesssim \mu^{\frac{1}{6}}\left(\frac{d}{\mu}\right)^{\frac{1}{4}}\|u\|_{F_{1}} \cdot\|v\|_{F_{\Omega, 1}} .
\end{aligned}
$$

This last expression can now be safely summed over both $\mu$ and $d$ to yield:

$$
\begin{aligned}
\text { (L.H.S.) (112) } & \lesssim \sum_{\substack{\mu, d \\
\mu \lesssim 1, d<c \mu}} \mu^{\frac{1}{6}}\left(\frac{d}{\mu}\right)^{\frac{1}{4}}\|u\|_{F_{1}} \cdot\|v\|_{F_{\Omega, 1}}, \\
& \lesssim\|u\|_{F_{1}} \cdot\|v\|_{F_{\Omega, 1}} .
\end{aligned}
$$

proof of estimate (113). We begin by fixing both $\mu$ and $d$, we use in order the angular decomposition (95) and orthogonality, the local Sobolev estimate (82), the 
Strichartz estimates (53) and (51), and finally the angular concentration estimate (88) to compute that:

$$
\begin{gathered}
\left\|S_{\mu, d}\left(S_{1, \bullet<\min \{d, c \mu\}} u \nabla S_{1, \bullet<\min \{d, c \mu\}} v\right)\right\|_{L^{2}\left(L^{2}\right)}, \\
\lesssim \quad\left(\sum_{\omega}\left\|S_{\mu, d}^{ \pm \omega}\left(B_{\left(\frac{d}{\mu}\right)^{\frac{1}{2}}}^{-\omega} S_{1, \bullet<\min \{d, c \mu\}} u \nabla B_{\left(\frac{d}{\mu}\right)^{\frac{1}{2}}}^{\omega} S_{1, \bullet<\min \{d, c \mu\}} v\right)\right\|_{L^{2}\left(L^{2}\right)}^{2}\right)^{\frac{1}{2}}, \\
\lesssim \quad \mu^{\frac{5}{12}} d^{\frac{1}{4}}\left(\sum_{\omega}\left\|B_{\left(\frac{d}{\mu}\right)^{\frac{1}{2}}}^{-\omega} S_{1, \bullet<\min \{d, c \mu\}} u \nabla B_{\left(\frac{d}{\mu}\right)^{\frac{1}{2}}}^{\omega} S_{1, \bullet<\min \{d, c \mu\}} v\right\|_{L^{2}\left(L^{\frac{3}{2}}\right)}^{2}\right)^{\frac{1}{2}}, \\
\lesssim \quad \mu^{\frac{5}{12}} d^{\frac{1}{4}} \sup _{\omega}\left\|B_{\left(\frac{d}{\mu}\right)^{\frac{1}{2}}}^{-\omega} S_{1, \bullet<\min \{d, c \mu\}} u\right\|_{L^{2}\left(L^{6}\right)} \\
\left.\lesssim \quad \mu^{\frac{5}{12}} d^{\frac{1}{4}} \sup _{\omega}\left\|B_{\left(\frac{d}{\mu}\right)^{\frac{1}{2}}}^{-\omega} u\right\|_{F_{1}} \cdot\left(\sum_{\omega}\left\|\nabla B_{\left(\frac{d}{\mu}\right)^{\frac{1}{2}}}^{\omega} S_{1, \bullet<\min \{d, c \mu\}} v\right\|_{L^{\infty}\left(L^{2}\right)}^{2}\right)^{\left.\frac{d}{\mu}\right)^{\frac{1}{2}}} v \|_{F_{1}}^{2}\right)^{\frac{1}{2}}, \\
\lesssim \quad \mu^{-\frac{1}{12}} d^{\frac{3}{4}}\|u\|_{F_{\Omega, 1}} \cdot\|v\|_{F_{1}} \cdot
\end{gathered}
$$

Multiplying this last expression through by $d^{-\frac{1}{2}}$ and summing over $d$ and $\mu$ yields:

$$
\begin{aligned}
\text { (L.H.S.) [113) } & \lesssim \sum_{\substack{\mu, d \\
\mu \lesssim 1, d \leqslant \mu}} \mu^{\frac{1}{6}}\left(\frac{d}{\mu}\right)^{\frac{1}{4}}\|u\|_{F_{\Omega, 1}} \cdot\|v\|_{F_{1}}, \\
& \lesssim\|u\|_{F_{\Omega, 1}} \cdot\|v\|_{F_{1}} .
\end{aligned}
$$

proof of estimate (114). This estimate is truly bilinear in nature, essentially due to the failure of the $L^{2}\left(L^{3}\right)$ endpoint version of (29). Therefore we must proceed in a more detailed fashion than the rest of the estimates in this section. For a fixed $\mu$ and $d$, we use the partial angular decomposition (94) in conjunction with the special multiplier bound (46), and the local Sobolev estimate (79) to compute that:

$$
\begin{aligned}
& \int\left(\sum_{\omega}\left\|S_{\mu, d}^{\omega}\left(S_{1, \bullet<\min \{d, c \mu\}} u \nabla S_{1, \bullet<\min \{d, c \mu\}} v\right)(t)\right\|_{L_{x}^{\infty}}^{2}\right)^{\frac{1}{2}} d t \\
& \lesssim \int\left(\sum_{\omega}\left\|B_{\left(\frac{d}{\mu}\right)^{\frac{1}{2}}}^{\omega} P_{\mu}\left(S_{1, \bullet<\min \{d, c \mu\}} u \nabla B_{\left(\frac{d}{\mu}\right)^{\frac{1}{2}}}^{\omega} S_{1, \bullet<\min \{d, c \mu\}} v\right)(t)\right\|_{L_{x}^{\infty}}^{2}\right)^{\frac{1}{2}} d t, \\
& \lesssim \quad \mu^{\frac{5}{4}-} d^{\frac{3}{4}-} \int\left(\sum_{\omega}\left\|\left(S_{1, \bullet<\min \{d, c \mu\}} u \nabla B_{\left(\frac{d}{\mu}\right)^{\frac{1}{2}}}^{\omega} S_{1, \bullet<\min \{d, c \mu\}} v\right)(t)\right\|_{L_{x}^{2+}}^{2}\right)^{\frac{1}{2}} d t .
\end{aligned}
$$

We now need to incorporate the "improved" Strichartz estimate (59). To do this, we begin by fixing $t$ and use the dual-scale Sobolev estimate from [5] along with 
Hölders inequality to compute that:

$$
\begin{gathered}
\left\|\left(S_{1, \bullet<\min \{d, c \mu\}} u \nabla B_{\left(\frac{d}{\mu}\right)^{\frac{1}{2}}}^{\omega} S_{1, \bullet<\min \{d, c \mu\}} v\right)(t)\right\|_{L_{x}^{2+}}, \\
\left.\lesssim \quad \mu^{2+}\left(\sum_{Q_{\alpha}} \| S_{1, \bullet<\min \{d, c \mu\}} u \nabla B_{\left(\frac{d}{\mu}\right)^{\frac{1}{2}}}^{\omega} S_{1, \bullet<\min \{d, c \mu\}} v\right)(t) \|_{L^{1}\left(Q_{\alpha}\right)}^{2+}\right)^{\frac{1}{2+}}, \\
\lesssim \quad \mu^{2+}\left(\sum_{Q_{\alpha}}\left\|S_{1, \bullet<\min \{d, c \mu\}} u(t)\right\|_{L^{2}\left(Q_{\alpha}\right)}^{3+}\right)^{\frac{1}{3+}} \cdot \\
\left(\sum_{Q_{\alpha}}\left\|\nabla B_{\left(\frac{d}{\mu}\right)^{\frac{1}{2}}}^{\omega} S_{1, \bullet<\min \{d, c \mu\}} v(t)\right\|_{L^{2}\left(Q_{\alpha}\right)}^{6}\right)^{\frac{1}{6}} .
\end{gathered}
$$

We now square sum this last expression with respect to $\omega$ and integrate with respect to time, followed by a use of Hölders inequality and the Strichartz estimates (59) and (58) to compute that:

(L.H.S.) 1311) ,

$$
\begin{aligned}
& \lesssim \quad \mu^{\frac{13}{4}-} d^{\frac{3}{4}-} \int\left(\sum_{Q_{\alpha}}\left\|S_{1, \bullet<\min \{d, c \mu\}} u(t)\right\|_{L^{2}\left(Q_{\alpha}\right)}^{3+}\right)^{\frac{1}{3+}} . \\
& \left(\sum_{\omega}\left(\sum_{Q_{\alpha}}\left\|\nabla B_{\left(\frac{d}{\mu}\right)^{\frac{1}{2}}}^{\omega} S_{1, \bullet<\min \{d, c \mu\}} v(t)\right\|_{L^{2}\left(Q_{\alpha}\right)}^{6}\right)^{\frac{1}{3}}\right)^{\frac{1}{2}} d t, \\
& \lesssim \quad \mu^{\frac{13}{4}-} d^{\frac{3}{4}-}\left\|\left(\sum_{Q_{\alpha}}\left\|S_{1, \bullet<\min \{d, c \mu\}} u(t)\right\|_{L^{2}\left(Q_{\alpha}\right)}^{3+}\right)^{\frac{1}{3+}}\right\|_{L_{t}^{2}} . \\
& \left(\sum_{\omega}\left\|\left(\sum_{Q_{\alpha}}\left\|\nabla B_{\left(\frac{d}{\mu}\right) \frac{1}{2}}^{\omega} S_{1, \bullet<\min \{d, c \mu\}} v(t)\right\|_{L^{2}\left(Q_{\alpha}\right)}^{6}\right)^{\frac{1}{6}}\right\|_{L_{t}^{2}}^{2}\right)^{\frac{1}{2}}, \\
& \lesssim \quad \mu^{\frac{7}{4}-} d^{\frac{3}{4}-}\|u\|_{F_{\Omega, 1}}\left(\sum_{\omega}\left\|B_{\left(\frac{d}{\mu}\right)^{\frac{1}{2}}}^{\omega} v\right\|_{F_{1}}^{2}\right)^{\frac{1}{2}}, \\
& \lesssim \quad \mu^{\frac{7}{4}-} d^{\frac{3}{4}-}\|u\|_{F_{\Omega, 1}}\|v\|_{F_{1}} .
\end{aligned}
$$

Multiplying this last expression by $\mu^{-\frac{3}{2}} d^{-\frac{1}{2}}$ and summing yields:

$$
\begin{aligned}
\text { (L.H.S.) 114) } & \lesssim \sum_{\substack{\mu, d: \\
\mu \lesssim 1, d \leqslant \mu}} \mu^{\frac{1}{2}-}\left(\frac{d}{\mu}\right)^{\frac{1}{4}-}\|u\|_{F_{\Omega, 1}}\|v\|_{F_{1}}, \\
& \lesssim\|u\|_{F_{\Omega, 1}}\|v\|_{F_{1}} .
\end{aligned}
$$


proof of estimates (115). We begin by fixing both $\mu$ and $d$, and using the angular decomposition (95) in conjunction with the special multiplier bound (45), the local Sobolev estimate (81), the angular concentration estimate (86), and finally the Strichartz estimate (57) to compute that:

$$
\begin{aligned}
& \int \sup _{\omega}\left\|S_{\mu, d}^{\omega}\left(S_{1, \bullet<\min \{d, c \mu\}} u \nabla S_{1, \bullet<\min \{d, c \mu\}} v\right)(t)\right\|_{L_{x}^{\infty}} d t, \\
& \lesssim \quad \int \sup _{\omega}\left\|B_{\left(\frac{d}{\mu}\right)^{\frac{1}{2}}}^{\omega} P_{\mu}\left(B_{\left(\frac{d}{\mu}\right)^{\frac{1}{2}}}^{-\omega} S_{1, \bullet<\min \{d, c \mu\}} u \nabla B_{\left(\frac{d}{\mu}\right)^{\frac{1}{2}}}^{\omega} S_{1, \bullet<\min \{d, c \mu\}} v\right)(t)\right\|_{L_{x}^{\infty}} d t, \\
& \lesssim \mu^{\frac{5}{3}-} d^{1-} \int \sup _{\omega}\left\|\left(B_{\left(\frac{d}{\mu}\right)^{\frac{1}{2}}}^{-\omega} S_{1, \bullet<\min \{d, c \mu\}} u \nabla B_{\left(\frac{d}{\mu}\right)^{\frac{1}{2}}}^{\omega} S_{1, \bullet<\min \{d, c \mu\}} v\right)(t)\right\|_{L_{x}^{\frac{3}{2}+}} d t \\
& \lesssim \mu^{\frac{5}{3}-} d^{1-} \int \sup _{\omega}\left(\left\|B_{\left(\frac{d}{\mu}\right)^{\frac{1}{2}}}^{-\omega} S_{1, \bullet<\min \{d, c \mu\}} u(t)\right\|_{L_{x}^{3+}}\right. \\
& \text { - } \left.\left\|\nabla B_{\left(\frac{d}{\mu}\right)^{\frac{1}{2}}}^{\omega} S_{1, \bullet<\min \{d, c \mu\}} v(t)\right\|_{L_{x}^{3+}}\right) d t, \\
& \lesssim \mu^{\frac{7}{6}-} d^{\frac{3}{2}-} \int\left\|S_{1, \bullet<\min \{d, c \mu\}}\langle\Omega\rangle^{\frac{1}{2}} u(t)\right\|_{L_{x}^{3+}} \cdot\left\|\nabla S_{1, \bullet<\min \{d, c \mu\}}\langle\Omega\rangle^{\frac{1}{2}} v(t)\right\|_{L_{x}^{3+}} d t, \\
& \lesssim \mu^{\frac{7}{6}-} d^{\frac{3}{2}-}\left\|S_{1, \bullet<\min \{d, c \mu\}}\langle\Omega\rangle^{\frac{1}{2}} u\right\|_{L^{2}\left(L^{3+}\right)} \cdot\left\|\nabla S_{1, \bullet<\min \{d, c \mu\}}\langle\Omega\rangle^{\frac{1}{2}} v\right\|_{L^{2}\left(L^{3+}\right)}, \\
& \lesssim \mu^{\frac{7}{6}-} d^{\frac{3}{2}-}\|u\|_{F_{\Omega, 1}} \cdot\|v\|_{F_{\Omega, 1}} .
\end{aligned}
$$

Multiplying this last expression through by $(\mu d)^{-1}$ and summing, we get that:

$$
\begin{aligned}
\text { (L.H.S.) [115) } & \lesssim \sum_{\substack{\mu, d: \\
\mu \lesssim 1, d \leqslant \mu}} \mu^{\frac{2}{3}-}\left(\frac{d}{\mu}\right)^{\frac{1}{2}-}\|u\|_{F_{\Omega, 1}} \cdot\|v\|_{F_{\Omega, 1}} \\
& \lesssim\|u\|_{F_{\Omega, 1}} \cdot\|v\|_{F_{\Omega, 1}} .
\end{aligned}
$$

\section{Inductive Estimates II. Low $\times$ High Frequency Interactions}

proof of estimate (117). The proof of this estimate follows directly from the boundedness of the $S_{\lambda}$ multiplier, Hölders inequality, and the Strichartz estimate (52) as well as the $L^{2}$ estimate (61):

$$
\begin{gathered}
\left\|S_{\lambda}\left(S_{1} u \nabla S_{\lambda, c \leqslant \bullet} v\right)\right\|_{L^{1}\left(L^{2}\right)}, \\
\lesssim c^{-\frac{1}{2}}\left\|S_{1} u\right\|_{L^{2}\left(L^{\infty}\right)} \cdot c^{\frac{1}{2}}\left\|\nabla S_{\lambda, c \leqslant \bullet} v\right\|_{L^{2}\left(L^{2}\right)}, \\
\lesssim c^{-\frac{1}{2}}\|u\|_{F_{1}} \cdot \lambda\|v\|_{F_{\lambda}} .
\end{gathered}
$$

For a fixed small $c$, this yields the desired result. 
proof of estimate (118). The proof here is a simple matter of Hölders inequality and the Strichartz estimates (52) and (55):

$$
\begin{array}{ll} 
& \sum_{c \leqslant d} d^{-\frac{1}{2}}\left\|S_{\lambda, d}\left(S_{1} u \nabla S_{\lambda, \bullet<c} v\right)\right\|_{L^{2}\left(L^{2}\right)}, \\
\lesssim & \sum_{c \leqslant d} d^{-\frac{1}{2}}\left\|S_{1} u\right\|_{L^{2}\left(L^{\infty}\right)} \cdot\left\|\nabla S_{\lambda, \bullet<c} v\right\|_{L^{\infty}\left(L^{2}\right)}, \\
\lesssim & \sum_{c \leqslant d} d^{-\frac{1}{2}}\|u\|_{F_{1}} \cdot \lambda\|v\|_{F_{\lambda}}, \\
\lesssim & \ln (c)\|u\|_{F_{1}} \cdot \lambda\|v\|_{F_{\lambda}} .
\end{array}
$$

For a fixed small $c$, this yields the desired result.

proof of estimate (119). For a fixed $d$ we use the decomposition (98) in conjunction with the multiplier bound (46), Hölders inequality, the local Sobolev estimate (76), and the Strichartz estimates (52) and (56) to prove that:

$$
\begin{gathered}
\int\left(\sum_{\omega}\left\|S_{\lambda, d}^{\omega}\left(S_{1} u \nabla S_{\lambda, \bullet<c} v\right)(t)\right\|_{L_{x}^{\infty}}^{2}\right)^{\frac{1}{2}} d t \\
\left.\vdots \quad \sum_{\substack{\omega_{1}, \omega_{2} \\
\left|\omega_{1}-\omega_{2}\right| \sim\left(\frac{d}{c \lambda}\right)^{\frac{1}{2}}}}\left\|S_{\lambda, d}^{\omega_{1}}\left(S_{1} u \nabla B_{\left(\frac{d}{c \lambda}\right)^{\frac{1}{2}}}^{\omega_{2}} S_{\lambda, \bullet<c} v\right)(t)\right\|_{L_{x}^{\infty}}^{2}\right)^{\frac{1}{2}} d t \\
\lesssim \quad c^{-\frac{3}{4}} \int\left(\sum_{\omega}\left\|\left(S_{1} u \nabla B_{\left(\frac{d}{c \lambda}\right)^{\frac{1}{2}}}^{\omega} S_{\lambda, \bullet<c} v\right)(t)\right\|_{L_{x}^{\infty}}^{2}\right)^{\frac{1}{2}} d t \\
\left.\lesssim \quad c^{-\frac{3}{4}} \int\left\|S_{1} u(t)\right\|_{L_{x}^{\infty}} \cdot\left(\sum_{\omega} \| \nabla B_{\left(\frac{d}{c \lambda}\right)^{\frac{1}{2}}}^{\omega} S_{\lambda, \bullet<c} v\right)(t) \|_{L_{x}^{\infty}}^{2}\right)^{\frac{1}{2}} d t \\
\lesssim \quad c^{-1} \lambda^{\frac{5}{12}} d^{\frac{1}{4}}\left\|S_{1} u\right\|_{L^{2}\left(L^{\infty}\right)} \cdot\left(\sum_{\omega}\left\|\nabla B_{\left(\frac{d}{c \lambda}\right)^{\frac{1}{2}}}^{\omega} S_{\lambda, \bullet<c} v\right\|_{L^{2}\left(L^{6}\right)}^{2}\right)^{\frac{1}{2}}, \\
\lesssim \quad c^{-1} \lambda^{\frac{15}{12}} d^{\frac{1}{4}}\|u\|_{F_{1}} \cdot \lambda\left(\sum_{\omega}\left\|B_{\left(\frac{d}{c \lambda}\right)^{\frac{1}{2}}}^{\omega} u\right\|_{F_{\lambda}}\right)^{\frac{1}{2}}, \\
\lesssim \quad c^{-1} \lambda^{\frac{15}{12}} d^{\frac{1}{4}}\|u\|_{F_{1}} \cdot \lambda\|v\|_{F_{\lambda}} \cdot
\end{gathered}
$$

Multiplying the last line above by the quantity $\lambda^{-\frac{3}{2}} d^{-\frac{1}{2}}$, using the fact that $1 \ll \lambda$, and summing over $d$ yields:

$$
\begin{aligned}
\text { (L.H.S.) 119) } & \lesssim \sum_{c \leqslant d} c^{-1} \lambda^{-\frac{1}{4}} d^{-\frac{1}{2}}\|u\|_{F_{1}} \cdot \lambda\|v\|_{F_{\lambda}}, \\
& \lesssim c^{-1} \ln (c)\|u\|_{F_{1}} \cdot \lambda\|v\|_{F_{\lambda}} .
\end{aligned}
$$

For a fixed small $c$, this yields the desired result. 
proof of estimate (120). For a fixed $d$ we use in order the decomposition (98), the multiplier bound (45), the local Sobolev estimate (78), the angular concentration estimate (86), and the Strichartz estimates (52) and (57) to compute that:

$$
\begin{aligned}
& \int \sup _{\omega}\left\|S_{\lambda, d}^{\omega}\left(S_{1} u \nabla S_{\lambda, \bullet<c} v\right)(t)\right\|_{L_{x}^{\infty}} d t, \\
& \lesssim \quad \int \sup _{\substack{\omega_{1}, \omega_{2} \\
\left|\omega_{1}-\omega_{2}\right| \sim\left(\frac{d}{c \lambda}\right)^{\frac{1}{2}}}}\left\|S_{\lambda, d}^{\omega_{1}}\left(S_{1} u \nabla B_{\left(\frac{d}{c \lambda}\right)^{\frac{1}{2}}}^{\omega_{2}} S_{\lambda, \bullet<c} v\right)(t)\right\|_{L_{x}^{\infty}} d t, \\
& \lesssim \quad \quad \quad \sup _{\omega}\left\|\left(S_{1} u \nabla B_{\left(\frac{d}{c \lambda}\right)^{\frac{1}{2}}}^{\omega} S_{\lambda, \bullet<c} v\right)(t)\right\|_{L_{x}^{\infty}} d t, \\
& \lesssim \quad \int\left\|S_{1} u(t)\right\|_{L_{x}^{\infty}} \cdot \sup _{\omega}\left\|\nabla B_{\left(\frac{d}{c \lambda}\right)^{\frac{1}{2}}}^{\omega} S_{\lambda, \bullet<c} v(t)\right\|_{L_{x}^{\infty}} d t, \\
& \lesssim \quad c^{\left(-\frac{1}{2}\right)+\lambda^{\frac{5}{6}-}} d^{\frac{1}{2}-} \int\left\|S_{1} u(t)\right\|_{L_{x}^{\infty}} \cdot \sup _{\omega}\left\|\nabla B_{\left(\frac{d}{c \lambda}\right)}^{\omega} S_{\lambda, \bullet<c} v(t)\right\|_{L_{x}^{3+}} d t \\
& \lesssim c^{\left(-\frac{3}{4}\right)+} \lambda^{\frac{7}{12}-} d^{\frac{3}{4}-} \int\left\|S_{1} u(t)\right\|_{L_{x}^{\infty}} \cdot\left\|\nabla\langle\Omega\rangle^{\frac{1}{2}} S_{\lambda, \bullet<c} v(t)\right\|_{L_{x}^{3+}} d t, \\
& \lesssim c^{\left(-\frac{3}{4}\right)+} \lambda^{\frac{7}{12}-} d^{\frac{3}{4}-}\left\|S_{1} u\right\|_{L^{2}\left(L^{\infty}\right)} \cdot\left\|\nabla\langle\Omega\rangle^{\frac{1}{2}} S_{\lambda, \bullet<c} v\right\|_{L^{2}\left(L^{3+}\right)}, \\
& \lesssim \quad c^{\left(-\frac{3}{4}\right)+} \lambda^{\frac{3}{4}+} d^{\frac{3}{4}-}\|u\|_{F_{1}} \cdot \lambda\|v\|_{F_{\Omega, \lambda}} \text {. }
\end{aligned}
$$

Multiplying this last line through by $(\lambda d)^{-1}$ and summing, while using the fact that $1 \ll \lambda$ yields:

$$
\begin{aligned}
\text { (L.H.S.) (120) } & \lesssim \sum_{c \leqslant d} c^{\left(-\frac{3}{4}\right)+} \lambda^{\left(-\frac{1}{4}\right)+} d^{-\left(\frac{1}{4}+\right)}\|u\|_{F_{1}} \cdot \lambda\|v\|_{F_{\Omega, \lambda}} \\
& \lesssim c^{-\frac{3}{4}}\|u\|_{F_{1}} \cdot \lambda\|v\|_{F_{\Omega, \lambda}}
\end{aligned}
$$

For a fixed $c$, this yields the desired result.

proof of estimate (122). For a fixed $d$, we use the angular decomposition (96), Hölders inequality, the local Sobolev estimate 75] and the concentration estimate (87), and finally the Strichartz estimate (53) and the $L^{2}$ estimate (60) to compute 
that:

$$
\begin{aligned}
& \int\left\|S_{\lambda, \bullet \leqslant d}\left(S_{1, \bullet \leqslant d} u \nabla S_{\lambda, d} v\right)(t)\right\|_{L_{x}^{2}} \\
& =\quad \int\left(\sum_{\omega}\left\|B_{d^{\frac{1}{2}}}^{\omega} P_{\lambda}\left(S_{1, \bullet \leqslant d}^{ \pm \omega} u \cdot \nabla B_{d^{\frac{1}{2}}}^{\omega} S_{\lambda, d} v\right)(t)\right\|_{L_{x}^{2}}^{2}\right)^{\frac{1}{2}} d t, \\
& \lesssim \quad \int\left(\sum_{\omega}\left\|S_{1, \bullet \leqslant d}^{ \pm \omega} u(t)\right\|_{L^{\infty}}^{2}\right)^{\frac{1}{2}} \cdot \sup _{\omega}\left\|\nabla B_{d^{\frac{1}{2}}}^{\omega} S_{\lambda, d} v(t)\right\|_{L_{x}^{2}} d t, \\
& \lesssim \quad d^{\frac{3}{4}} \int\left(\sum_{\omega}\left\|S_{1, \bullet \leqslant d}^{ \pm \omega} u(t)\right\|_{L^{6}}^{2}\right)^{\frac{1}{2}} \cdot\left\|\nabla S_{\lambda, d}\langle\Omega\rangle v(t)\right\|_{L_{x}^{2}} d t, \\
& \lesssim \quad d^{\frac{1}{4}}\left(\sum_{\omega}\left\|S_{1, \bullet \leqslant d}^{ \pm \omega} u\right\|_{L^{2}\left(L^{6}\right)}^{2}\right)^{\frac{1}{2}} \cdot d^{\frac{1}{2}}\left\|\nabla S_{\lambda, d}\langle\Omega\rangle v\right\|_{L^{2}\left(L^{2}\right)}, \\
& \lesssim \quad d^{\frac{1}{4}}\|u\|_{F_{1}} \cdot \lambda\|v\|_{F_{\Omega, \lambda}} .
\end{aligned}
$$

This last expression can now be safely summed over $d$ to yield:

$$
\begin{aligned}
\text { (L.H.S.) (122) } & \lesssim \sum_{d \leqslant 1} d^{\frac{1}{4}}\|u\|_{F_{1}} \cdot \lambda\|v\|_{F_{\Omega, \lambda}}, \\
& \lesssim\|u\|_{F_{1}} \cdot \lambda\|v\|_{F_{\Omega, \lambda}} .
\end{aligned}
$$

proof of estimate (123). Here, for a fixed $d$, we use in order the angular decomposition (96), Hölders inequality, the local Sobolev estimate (77), the concentration estimate (86), and the Strichartz estimate (54) as well as the $L^{2}$ estimate (60) to compute that:

$$
\begin{array}{cc} 
& \int\left\|S_{\lambda, \bullet \leqslant d}\left(S_{1, \bullet \leqslant d} u \cdot \nabla S_{\lambda, d} v\right)(t)\right\|_{L_{x}^{2}} \\
= & \int\left(\sum_{\omega}\left\|B_{d^{\frac{1}{2}}}^{\omega} P_{\lambda}\left(S_{1, \bullet \leqslant d}^{ \pm \omega} u \cdot \nabla B_{d^{\frac{1}{2}}}^{\omega} S_{\lambda, d} v\right)(t)\right\|_{L_{x}^{2}}^{2}\right)^{\frac{1}{2}} d t, \\
\lesssim & \quad \int \sup _{\omega}\left\|S_{1, \bullet \leqslant d}^{ \pm \omega} u(t)\right\|_{L^{\infty}} \cdot\left(\sum_{\omega}\left\|\nabla B_{d^{\frac{1}{2}}}^{\omega} S_{\lambda, d} v(t)\right\|_{L_{x}^{2}}^{2}\right)^{\frac{1}{2}} d t, \\
\lesssim & d^{\frac{1}{2}-} \quad \int \sup _{\omega}\left\|S_{1, \bullet \leqslant d}^{ \pm \omega} u(t)\right\|_{L^{3+}} \cdot\left\|\nabla S_{\lambda, d} v(t)\right\|_{L_{x}^{2}} d t, \\
\lesssim & d^{\frac{3}{4}-} \quad \int\left\|S_{1, \bullet \leqslant d}\langle\Omega\rangle^{\frac{1}{2}} u(t)\right\|_{L^{3+}} \cdot\left\|\nabla S_{\lambda, d} v(t)\right\|_{L_{x}^{2}} d t, \\
\lesssim & d^{\frac{1}{4}-}\left\|S_{1, \bullet \leqslant d}\langle\Omega\rangle^{\frac{1}{2}} u\right\|_{L^{2}\left(L^{3+}\right)} \cdot d^{\frac{1}{2}}\left\|\nabla S_{\lambda, d} v\right\|_{L^{2}\left(L^{2}\right)}, \\
\lesssim & d^{\frac{1}{4}-}\|u\|_{F_{\Omega, 1}} \cdot \lambda\|v\|_{F_{\lambda}} .
\end{array}
$$


This last expression can now be safely summed over $d$ to yield:

$$
\begin{aligned}
\text { (L.H.S.) (123) } & \lesssim \sum_{d \leqslant 1} d^{\frac{1}{4}-}\|u\|_{F_{\Omega, 1}} \cdot \lambda\|v\|_{F_{\lambda}}, \\
& \lesssim\|u\|_{F_{\Omega, 1}} \cdot \lambda\|v\|_{F_{\lambda}} .
\end{aligned}
$$

proof of estimate (124). The proof here uses essentially the same steps as (122) above. We begin by fixing $d$ and use the angular decomposition (96), Hölders inequality and the local Sobolev estimate (75), the Strichartz estimates (53) and (55), and the concentration estimate (88) to compute that:

$$
\begin{array}{cc} 
& \left\|S_{\lambda, d}\left(S_{1, \bullet \leqslant d} u \cdot \nabla S_{\lambda, \bullet<d} v\right)\right\|_{L^{2}\left(L^{2}\right)}, \\
\lesssim & \left(\sum_{\omega}\left\|B_{d^{\frac{1}{2}}}^{\omega} S_{\lambda, d}\left(S_{1, \bullet<d}^{ \pm \omega} u \cdot \nabla B_{d^{\frac{1}{2}}}^{\omega} S_{\lambda, \bullet<d} v\right)\right\|_{L^{2}\left(L^{2}\right)}^{2}\right)^{\frac{1}{2}}, \\
\lesssim \quad & d^{\frac{1}{4}}\left(\sum_{\omega}\left\|S_{1, \bullet \leqslant d}^{ \pm \omega} u\right\|_{L^{2}\left(L^{6}\right)}^{2}\right)^{\frac{1}{2}} \cdot \sup _{\omega}\left\|\nabla B_{d^{\frac{1}{2}}}^{\omega} S_{\lambda, \bullet<d} v\right\|_{L^{\infty}\left(L^{2}\right)}, \\
\lesssim \quad & d^{\frac{1}{4}}\left(\sum_{\omega}\left\|B_{d^{\frac{1}{2}}}^{\omega} u\right\|_{F_{1}}^{2}\right)^{\frac{1}{2}} \cdot \lambda \sup _{\omega}\left\|B_{d^{\frac{1}{2}}}^{\omega} v\right\|_{F_{\lambda}}, \\
\lesssim \quad & d^{\frac{3}{4}}\|u\|_{F_{1}} \cdot \lambda\|v\|_{F_{\Omega, \lambda}} .
\end{array}
$$

We can now multiply this last expression by $d^{-\frac{1}{2}}$ and sum to yield:

$$
\begin{aligned}
\text { (L.H.S.) (124) } & \lesssim \sum_{d \leqslant 1} d^{\frac{1}{4}}\|u\|_{F_{1}} \cdot \lambda\|v\|_{F_{\Omega, \lambda}}, \\
& \lesssim\|u\|_{F_{1}} \cdot \lambda\|v\|_{F_{\Omega, \lambda}} .
\end{aligned}
$$

proof of estimate (125). The proof here uses essentially the same steps as (123) above. For fixed $d$ we use in order the angular decomposition (96), Hölders inequality and the local Sobolev estimate (77), the concentration estimate (86), and the Strichartz estimates (54) and (55) to compute that:

$$
\begin{array}{cc} 
& \left\|S_{\lambda, d}\left(S_{1, \bullet \leqslant d} u \cdot \nabla S_{\lambda, \bullet<d} v\right)\right\|_{L^{2}\left(L^{2}\right)}, \\
\lesssim & \left(\sum_{\omega}\left\|B_{d^{\frac{1}{2}}}^{\omega} S_{\lambda, d}\left(S_{1, \bullet \leqslant d}^{ \pm \omega} u \cdot \nabla B_{d^{\frac{1}{2}}}^{\omega} S_{\lambda, \bullet<d} v\right)\right\|_{L^{2}\left(L^{2}\right)}^{2}\right)^{\frac{1}{2}}, \\
\lesssim & d^{\frac{1}{2}-} \sup _{\omega}\left\|S_{1, \bullet \leqslant d}^{ \pm \omega} u\right\|_{L^{2}\left(L^{3+}\right)} \cdot\left(\sum_{\omega}\left\|\nabla B_{d^{\frac{1}{2}}}^{\omega} S_{\lambda, \bullet<d} v\right\|_{L^{\infty}\left(L^{2}\right)}^{2}\right)^{\frac{1}{2}}, \\
\lesssim & d^{\frac{3}{4}-}\left\|S_{1, \bullet \leqslant d}^{ \pm \omega}\langle\Omega\rangle^{\frac{1}{2}} u\right\|_{L^{2}\left(L^{3+}\right)} \cdot\left(\sum_{\omega}\left\|\nabla B_{d^{\frac{1}{2}}}^{\omega} S_{\lambda, \bullet<d} v\right\|_{L^{\infty}\left(L^{2}\right)}^{2}\right)^{\frac{1}{2}}, \\
\lesssim \quad & d^{\frac{3}{4}-}\|u\|_{F_{\Omega, 1}} \cdot \lambda\|v\|_{F_{\lambda}} .
\end{array}
$$


We can now multiply this last expression by $d^{-\frac{1}{2}}$ and sum to yield:

$$
\begin{aligned}
\text { (L.H.S.) } & \lesssim \sum_{d \leqslant 1} d^{\frac{1}{4}-}\|u\|_{F_{\Omega, 1}} \cdot \lambda\|v\|_{F_{\lambda}}, \\
& \lesssim\|u\|_{F_{\Omega, 1}} \cdot \lambda\|v\|_{F_{\lambda}} .
\end{aligned}
$$

proof of estimate (126). For a fixed $d$ we use the angular decomposition (97) in conjunction with the multiplier bound (65), the multiplier Lemma 4.1 and Hölders inequality, the local Sobolev lemmas (75) and (78), the angular concentration estimate (86), and finally the Strichartz estimates (53) and (57) to compute that:

$$
\begin{aligned}
& \int\left(\sum_{\substack{\theta: \\
|\theta| \sim d^{\frac{1}{2}}}} \sup _{\omega \subseteq \theta}\left\|S_{\lambda, \bullet<\min \{d, c\}}^{\omega}\left(S_{1, d} u \nabla S_{\lambda, \bullet<\min \{d, c\}} v\right)(t)\right\|_{L_{x}^{\infty}}^{2}\right)^{\frac{1}{2}} d t, \\
= & \int\left(\sum_{\substack{\theta: \\
|\theta| \sim d^{\frac{1}{2}}}} \sup _{\omega \subseteq \theta}\left\|B_{\left(\frac{d}{\lambda}\right)^{\frac{1}{2}}}^{\omega} P_{\lambda}\left(S_{1, d}^{ \pm \theta} u \nabla S_{\lambda, \bullet<\min \{d, c\}}^{\omega} v\right)(t)\right\|_{L_{x}^{\infty}}^{2}\right)^{\frac{1}{2}} d t \\
\lesssim \quad & \left.\int\left(\sum_{\theta}\left\|S_{1, d}^{ \pm \theta} u(t)\right\|_{L_{x}^{\infty}}^{2} \cdot \sup _{\omega \subseteq \theta} \| \nabla S_{\lambda, \bullet<\min \{d, c\}}^{\omega} v\right)(t) \|_{L_{x}^{\infty}}^{2}\right)^{\frac{1}{2}} d t \\
\lesssim \quad \lambda^{\frac{5}{6}-} d^{\frac{3}{4}-} & \int\left(\sum_{\theta}\left\|S_{1, d}^{ \pm \theta} u(t)\right\|_{L_{x}^{6}}^{2}\right)^{\frac{1}{2}} \cdot \sup _{\omega}\left\|\nabla S_{\lambda, \bullet<\min \{d, c\}}^{\omega} v(t)\right\|_{L_{x}^{3+}} d t \\
\lesssim \quad \lambda^{\frac{7}{12}}-d^{1-} & \int\left(\sum_{\theta}\left\|S_{1, d}^{ \pm \theta} u(t)\right\|_{L_{x}^{6}}^{2}\right)^{\frac{1}{2}} \cdot\left\|\nabla S_{\lambda, \bullet<\min \{d, c\}}\langle\Omega\rangle^{\frac{1}{2}} v(t)\right\|_{L_{x}^{3+}} d t \\
\lesssim \quad \lambda^{\frac{3}{4}+} d^{1-} & \|u\|_{F_{1}} \cdot \lambda\|v\|_{F_{\Omega, \lambda}} \cdot
\end{aligned}
$$

Multiplying this last expression through by $\left(\lambda d^{\frac{1}{2}}\right)^{-1}$ and summing over $d$ yields:

$$
\begin{aligned}
\text { (L.H.S.) [126) } & \lesssim \sum_{d \leqslant 1} d^{\frac{1}{4}-}\left(\frac{d}{\lambda}\right)^{\frac{1}{4}-}\|u\|_{F_{1}} \cdot \lambda\|v\|_{F_{\Omega, \lambda}}, \\
& \lesssim\|u\|_{F_{1}} \cdot \lambda\|v\|_{F_{\Omega, \lambda}} .
\end{aligned}
$$

proof of estimate (127). We begin by fixing $d$ and use the angular decomposition (97) in conjunction with the multiplier bound (46) and Hölders inequality, the local Sobolev estimates (75) and (78), the angular concentration estimate (86), and the 
Strichartz estimates (53) and (57) to compute that:

$$
\begin{aligned}
& \int\left(\sum_{\omega}\left\|S_{\lambda, \bullet<\min \{d, c\}}^{\omega}\left(S_{1, d} u \nabla S_{\lambda, \bullet<\min \{d, c\}} v\right)(t)\right\|_{L_{x}^{\infty}}^{2}\right)^{\frac{1}{2}} d t, \\
& \lesssim \int\left(\sum_{\substack{\omega_{1, \omega_{2}}: \\
\left|\omega_{1} \mp \omega_{2}\right| \sim d^{\frac{1}{2}}}}\left\|S_{1, d}^{ \pm \omega_{2}} u(t)\right\|_{L_{x}^{\infty}}^{2} \cdot\left\|\nabla S_{\lambda, \bullet \min \{d, c\}}^{\omega_{1}} v(t)\right\|_{L_{x}^{\infty}}^{2}\right)^{\frac{1}{2}} d t, \\
& \lesssim \quad \lambda^{\frac{5}{12}} d^{\frac{3}{4}-} \int \sup _{\omega}\left\|S_{1, d}^{ \pm \omega} u(t)\right\|_{L_{x}^{3+}} \cdot\left(\sum_{\omega}\left\|\nabla S_{\lambda, \bullet \min \{d, c\}}^{\omega} v(t)\right\|_{L_{x}^{6}}^{2}\right)^{\frac{1}{2}} d t, \\
& \lesssim \quad \lambda^{\frac{5}{12}} d^{1-}\left\|S_{1, d}\langle\Omega\rangle^{\frac{1}{2}} u\right\|_{L^{2}\left(L^{3+}\right)} \cdot\left(\sum_{\omega}\left\|\nabla S_{\lambda, \bullet<\min \{d, c\}}^{\omega} v\right\|_{L^{2}\left(L^{6}\right)}^{2}\right)^{\frac{1}{2}}, \\
& \lesssim \quad \lambda^{\frac{15}{12}} d^{1-}\|u\|_{F_{\Omega, 1}} \cdot \lambda\|v\|_{F_{\lambda}} \cdot
\end{aligned}
$$

Multiplying this last expression through by $\left(\lambda^{\frac{3}{2}} d^{\frac{1}{2}}\right)^{-1}$ and summing over $d$ yields:

$$
\begin{aligned}
\text { (L.H.S.) } & \lesssim \sum_{d \leqslant 1} d^{\frac{1}{4}-}\left(\frac{d}{\lambda}\right)^{\frac{1}{4}}\|u\|_{F_{\Omega, 1}} \cdot \lambda\|v\|_{F_{\lambda}} \\
& \lesssim\|u\|_{F_{\Omega, 1}} \cdot \lambda\|v\|_{F_{\lambda}} .
\end{aligned}
$$

proof of estimate (128). Fixing $d$ we use in order the angular decomposition (97) in conjunction with the multiplier bound (45) and Hölders inequality, the local Sobolev estimates (77) and (78), the angular concentration estimate (86), and the Strichartz estimates (54) and (57) to compute that:

$$
\begin{aligned}
& \int \sup _{\omega}\left\|S_{\lambda, \bullet<\min \{d, c\}}^{\omega}\left(S_{1, d} u \nabla S_{\lambda, \bullet<\min \{d, c\}} v\right)(t)\right\|_{L_{x}^{\infty}} d t, \\
& \lesssim \quad \int \sup _{\omega}\left\|S_{1, d}^{ \pm \omega} u(t)\right\|_{L_{x}^{\infty}} \cdot \sup _{\omega}\left\|\nabla S_{\lambda, \bullet<\min \{d, c\}}^{\omega} v(t)\right\|_{L_{x}^{\infty}} d t, \\
& \lesssim \quad \lambda^{\frac{5}{6}-} d^{1-} \int \sup _{\omega}\left\|S_{1, d}^{ \pm \omega} u(t)\right\|_{L_{x}^{3+}} \cdot \sup _{\omega}\left\|\nabla S_{\lambda, \bullet<\min \{d, c\}}^{\omega} v(t)\right\|_{L_{x}^{3+}} d t, \\
& \lesssim \quad \lambda^{\frac{7}{12}-} d^{\frac{3}{2}-}\left\|S_{1, d}\langle\Omega\rangle^{\frac{1}{2}} u\right\|_{L^{2}\left(L^{3+}\right)} \cdot\left\|\nabla S_{\lambda, \bullet<\min \{d, c\}}\langle\Omega\rangle^{\frac{1}{2}} v\right\|_{L^{2}\left(L^{3+}\right)}, \\
& \lesssim \quad \lambda^{\frac{3}{4}+} d^{\frac{3}{2}-}\|u\|_{F_{\Omega, 1}} \cdot \lambda\|v\|_{F_{\Omega, \lambda}} .
\end{aligned}
$$

Multiplying this last expression through by $(\lambda d)^{-1}$ and summing over yields:

$$
\begin{aligned}
\text { (L.H.S.) } & \lesssim \sum_{d \leqslant 1} d^{\frac{1}{4}-}\left(\frac{d}{\lambda}\right)^{\frac{1}{4}-}\|u\|_{F_{\Omega, 1}} \cdot \lambda\|v\|_{F_{\Omega, \lambda}} \\
& \lesssim\|u\|_{F_{\Omega, 1}} \cdot \lambda\|v\|_{F_{\Omega, \lambda}} .
\end{aligned}
$$


proof of estimate (129). For any decomposition $u=\sum_{\alpha} u^{\alpha}$, we begin by fixing $d$ and using the angular decomposition (96) in conjunction with the multiplier Lemma 4.1 and Hölders inequality to compute that:

$$
\begin{gathered}
\int\left\|S_{\lambda, \bullet<\min \{d, c\}}\left(S_{1, d} u \cdot \nabla S_{\lambda, \bullet<\min \{d, c\}} v\right)(t)\right\|_{L_{x}^{2}} d t, \\
\lesssim \quad \sum_{\alpha} \int\left\|S_{\lambda, \bullet<\min \{d, c\}}\left(S_{1, d} u^{\alpha} \cdot \nabla S_{\lambda, \bullet<\min \{d, c\}} v\right)(t)\right\|_{L_{x}^{2}} d t, \\
\lesssim \quad \sum_{\alpha} \int\left(\sum_{\omega}\left\|B_{d^{\frac{1}{2}}}^{\omega} P_{\lambda}\left(S_{1, d}^{ \pm \omega} u^{\alpha} \cdot \nabla B_{d^{\frac{1}{2}}}^{\omega} S_{\lambda, \bullet<\min \{d, c\}} v\right)(t)\right\|_{L_{x}^{2}}^{2}\right)^{\frac{1}{2}} d t, \\
\lesssim \quad \sum_{\alpha} \int\left(\sum_{\omega}\left\|S_{1, d}^{ \pm \omega} u^{\alpha}(t)\right\|_{L_{x}^{\infty}}^{2} \cdot\left\|\nabla B_{d^{\frac{1}{2}}}^{\omega} S_{\lambda, \bullet<\min \{d, c\}} v(t)\right\|_{L_{x}^{2}}^{2}\right)^{\frac{1}{2}} d t .
\end{gathered}
$$

Now, for each fixed $\alpha$, we let $\left\{\theta_{\alpha, d}\right\}$ be a collection of angles such that $|\omega| \leqslant\left|\theta_{\alpha, d}\right|$. Then with a repeated use of Hölders inequality, the concentration estimate [87], and the Strichartz estimate (55), we see that:

(L.H.S.) 132) for a fixed $\alpha$,

$$
\begin{aligned}
& \lesssim\left(\sum_{\theta_{\alpha, d}} \sup _{\omega \subseteq \theta_{\alpha, d}}\left\|S_{1, d}^{ \pm \omega} u^{\alpha}(t)\right\|_{L_{x}^{\infty}}^{2} \cdot \sum_{\omega \subseteq \theta_{\alpha, d}}\left\|\nabla B_{d^{\frac{1}{2}}}^{\omega} S_{\lambda, \bullet<\min \{d, c\}} v(t)\right\|_{L_{x}^{2}}^{2}\right)^{\frac{1}{2}} d t, \\
& \lesssim \int\left(\sum_{\theta_{\alpha, d}} \sup _{\omega \subseteq \theta_{\alpha, d}}\left\|S_{1, d}^{ \pm \omega} u^{\alpha}(t)\right\|_{L_{x}^{\infty}}^{2}\right)^{\frac{1}{2}} \cdot \sup _{\theta_{\alpha, d}}\left\|\nabla B_{\left|\theta_{\alpha, d}\right|}^{\theta_{\alpha, d}} S_{\lambda, \bullet<\min \{d, c\}} v(t)\right\|_{L_{x}^{2}} d t, \\
& \lesssim\left|\theta_{\alpha, d}\right| \int\left(\sum_{\theta_{\alpha, d}} \sup _{\omega \subseteq \theta_{\alpha, d}}\left\|S_{1, d}^{ \pm \omega} u^{\alpha}(t)\right\|_{L_{x}^{\infty}}^{2}\right)^{\frac{1}{2}} \cdot\left\|\nabla S_{\lambda, \bullet \min \{d, c\}}\langle\Omega\rangle v(t)\right\|_{L_{x}^{2}} d t, \\
& \lesssim\left|\theta_{\alpha, d}\right| \int\left(\sum_{\theta_{\alpha, d}} \sup _{\omega \subseteq \theta_{\alpha, d}}\left\|S_{1, d}^{ \pm \omega} u^{\alpha}(t)\right\|_{L_{x}^{\infty}}^{2}\right)^{\frac{1}{2}} d t \cdot\left\|\nabla S_{\lambda, \bullet<\min \{d, c\}}\langle\Omega\rangle v\right\|_{L^{\infty}\left(L^{2}\right)}, \\
& \lesssim\left|\theta_{\alpha, d}\right| \int\left(\sum_{\theta_{\alpha, d}} \sup _{\omega \subseteq \theta_{\alpha, d}}\left\|S_{1, d}^{ \pm \omega} u^{\alpha}(t)\right\|_{L_{x}^{\infty}}^{2}\right)^{\frac{1}{2}} d t \cdot \lambda\|v\|_{F_{\Omega, \lambda}} .
\end{aligned}
$$

Finally, assuming that $\left\{\theta_{\alpha, d}\right\}$ and the $u^{\alpha}$ minimize the $Z_{1}$ norm for this particular $u$, we get that:

$$
\begin{aligned}
\text { (L.H.S.) [129) } & \lesssim \sum_{\alpha, d}\left|\theta_{\alpha, d}\right| \int\left(\sum_{\theta_{\alpha, d}} \sup _{\omega \subseteq \theta_{\alpha, d}}\left\|S_{1, d}^{ \pm \omega} u^{\alpha}(t)\right\|_{L_{x}^{\infty}}^{2}\right)^{\frac{1}{2}} d t \cdot \lambda\|v\|_{F_{\Omega, \lambda}}, \\
& \lesssim\|v\|_{Z_{1}} \cdot \lambda\|v\|_{F_{\Omega, \lambda}} .
\end{aligned}
$$


proof of estimate (130). Fixing $d$ we use the angular decomposition (96) in conjunction with the multiplier Lemma 4.1 several rounds of Hölders inequality, and the Strichartz estimate (55) to compute that:

$$
\begin{aligned}
& \int\left\|S_{\lambda, \bullet<\min \{d, c\}}\left(S_{1, d} u \nabla S_{\lambda, \bullet<\min \{d, c\}} v\right)(t)\right\|_{L_{x}^{2}} d t, \\
\lesssim & \int\left(\sum_{\omega}\left\|S_{1, d}^{ \pm \omega} u(t)\right\|_{L_{x}^{\infty}}^{2} \cdot\left\|\nabla B_{d^{\frac{1}{2}}}^{\omega} S_{\lambda, \bullet<\min \{d, c\}} v(t)\right\|_{L_{x}^{2}}^{2}\right)^{\frac{1}{2}} d t, \\
\lesssim \quad & \int \sup _{\omega}\left\|S_{1, d}^{ \pm \omega} u(t)\right\|_{L_{x}^{\infty}} \cdot\left(\sum_{\omega}\left\|\nabla B_{d^{\frac{1}{2}}}^{\omega} S_{\lambda, \bullet<\min \{d, c\}} v(t)\right\|_{L_{x}^{2}}^{2}\right)^{\frac{1}{2}} d t, \\
\lesssim \quad & \int \sup _{\omega}\left\|S_{1, d}^{ \pm \omega} u(t)\right\|_{L_{x}^{\infty}} d t \cdot\left\|\nabla S_{\lambda, \bullet<\min \{d, c\}} v\right\|_{L^{\infty}\left(L^{2}\right)}, \\
\lesssim \quad & \int \sup _{\omega}\left\|S_{1, d}^{ \pm \omega} u(t)\right\|_{L_{x}^{\infty}} d t \cdot \lambda\|v\|_{F_{\lambda}} .
\end{aligned}
$$

Summing this last expression over $d$ yields:

$$
\begin{aligned}
\text { (L.H.S.) [130) } & \lesssim \sum_{d} \int \sup _{\omega}\left\|S_{1, d}^{ \pm \omega} u(t)\right\|_{L_{x}^{\infty}} d t \cdot \lambda\|v\|_{F_{\lambda}} \\
& \lesssim\|v\|_{Z_{\Omega, 1}} \cdot \lambda\|v\|_{F_{\lambda}} .
\end{aligned}
$$

\section{REFERENCES}

[1] Lars Hörmander On the fully nonlinear Cauchy problem with small data. II. Microlocal analysis and nonlinear waves (Minneapolis, MN, 1988-1989), 51-81, IMA Vol. Math. Appl., 30, Springer, New York, 1991.

[2] Markus Keel, Terence Tao Endpoint Strichartz estimates. Amer. J. Math. 120 (1998), no. 5, 955-980.

[3] Sergiu Klainerman Uniform decay estimates and the Lorentz invariance of the classical wave equation. Comm. Pure Appl. Math. 38 (1985), no. 3, 321-332.

[4] Sergiu Klainerman, Matei Machedon Estimates for null forms and the spaces $H^{s, \delta}$. Internat. Math. Res. Notices 1996, no. 17, 853-865.

[5] Sergiu Klainerman, Igor Rodnianski, Terence Tao A physical space approach to wave equation bilinear estimates. Dedicated to the memory of Thomas H. Wolff. J. Anal. Math. 87 (2002), 299-336.

[6] Sergiu Klainerman, Sigmund Selberg, Bilinear estimates and applications to nonlinear wave equations. Commun. Contemp. Math. 4 (2002), no. 2, 223-295.

[7] Sergiu Klainerman, Daniel Tataru On the optimal local regularity for Yang-Mills equations in $R^{4+1}$. J. Amer. Math. Soc. 12 (1999), no. 1, 93-116.

[8] Hans Lindblad Counterexamples to local existence for semi-linear wave equations. Amer. J. Math. 118 (1996), no. 1, 1-16.

[9] Matei Machedon, Jacob Sterbenz Almost optimal local well-posedness for the (3+1)dimensional Maxwell-Klein-Gordon equations. to appear in JAMS

[10] Elias Stein Singular integrals and differentiability properties of functions. Princeton Mathematical Series, No. 30 Princeton University Press, Princeton, N.J. 1970.

[11] Jacob Sterbenz Global Regularity for General Non-Linear Wave Equations I. $(6+1)$ and Higher Dimensions. preprint

[12] Jacob Sterbenz Angular Regularity and Strichartz Estimates for the Wave Equation. preprint 
[13] Terence Tao Low regularity semi-linear wave equations. Comm. Partial Differential Equations 24 (1999), no. 3-4, 599-629.

[14] Daniel Tataru On the equation $\square u=|\nabla u|^{2}$ in $5+1$ dimensions. Math. Res. Lett. 6 (1999), no. 5-6, 469-485.

Department of Mathematics, Princeton University, Princeton, NJ 08540

E-mail address: sterbenz@math.princeton.edu 